\title{
1 A Portable and Wide Energy Range Semiconductor-Based Neutron Spectrometer
}

2 C.B. Hoshor ${ }^{a^{*}}$, T.M. Oakes ${ }^{b}$, E.R. Myers ${ }^{a}$, B.J. Rogers, ${ }^{a}$, J.E. Currie ${ }^{a}$, S.M. Young, J. A. Crow ${ }^{a}$, P.R. Scott ${ }^{a}$,

3 W.H. Miller ${ }^{b, c}$, S. L. Bellinger ${ }^{d}$, T.J. Sobering ${ }^{d}$, R.G. Fronk ${ }^{d}$, J.K. Shultis ${ }^{d}$, D.S. McGregor ${ }^{d}$, A.N. Caruso ${ }^{a *}$

$4 \quad{ }^{a}$ Department of Physics, University of Missouri - Kansas City

$5 \quad$ buclear Science and Engineering Institute, University of Missouri - Columbia

6 'Missouri University Research Reactor, Columbia, MO

$7 \quad$ Department of Mechanical and Nuclear Engineering, Kansas State University, Manhattan, KS

[Keywords: neutron spectrometer, solid-state neutron detector, neutron source identification, portable neutron detector, moderating spectrometer, semiconductor-based neutron detector]

\section{Abstract}

Hand-held instruments with the ability to passively detect and identify sources of neutron radiationeither bare or obscured by neutron moderating and/or absorbing material(s) -in real time are of interest in a variety of non-proliferation and health physics applications. Such an instrument must provide a means to high intrinsic detection efficiency and energy-sensitive measurements of free neutron fields, for neutrons ranging from thermal energies to the top end of the evaporation spectrum. To address and overcome the challenges inherent to the aforementioned applications, four solid-state moderating-type neutron spectrometers of varying cost, weight, and complexity have been designed, fabricated, and tested. The motivation of this work is to introduce these novel human-portable instruments by discussing the fundamental theory of their operation, investigating and analyzing the principal considerations for optimal instrument design, and evaluating the capability of each of the four fabricated spectrometers to meet the application needs.

Version: 31 JULY 2015

*Corresponding Author: Anthony N. Caruso, carusoan@umkc.edu, 257 Flarsheim Hall, 5110 Rockhill 


\section{Introduction}

32 Portable instruments that can detect and identify neutron sources by high intrinsic efficiency and energy-sensitive measurement of free neutrons, ranging from thermal energies ( $0.025 \mathrm{meV}$ ) to top end of the evaporation spectrum ( $20 \mathrm{MeV})$, are important to applications in non-proliferation and health physics. For non-proliferation applications, the goal is a sensitivity and spectroscopic resolution that will allow for unknown source detection and identification from neutrons alone as a crude complement to portable gamma-ray spectroscopic instruments; detection over the large energy range is important for sources in unknown moderating/absorbing configurations. In health physics applications, the neutron dose equivalent rises and falls more than two-orders of magnitude between the thermal and 14-MeV energy range, presenting substantial dosimetry challenges. In commercially available rem meters [1] the average dose equivalent error is significant due to poor energy sensitivity in the upper end of this large range. To address the challenges inherent to these applications, this work presents and compares the empirical and computational design and results of a new class of real-time human-portable neutron spectrometer, building from the foundation established by Bonner [2], and enabled by the high thermal neutron detection efficiency density (thermal neutron detection efficiency per unit active volume) and spatial resolution of semiconductor-based neutron detectors [3].

\section{$47 \quad 2 . \quad$ Instrument Design}

\subsection{Background}

The instrument geometries, detector-moderator configurations, analysis methods, and results described here are a synthesis of, and improvement on, the multi-sphere- and long-counter-based moderatingtype neutron spectrometers [2, 4]. The major deficiencies in the multi-sphere spectrometers are: (a) the inability to measure neutron intensity with each sphere radius simultaneously, (b) the poor spatial resolution of detected thermalized neutrons, (c) the very low intrinsic neutron detection efficiency, and (d) the unportable mass of the near largest to largest radius moderating sphere used to measure neutron intensity at the top end of the fast neutron energy range (i.e., a $30-\mathrm{cm}$ radius). In the long counter design, neutron intensity is measured axially in a cylindrical geometry, replacing the radial dependence of the measured neutron intensity in the spherical geometry. Although the cylindrical geometry prevents equal sensitivity over $4 \pi$, an instrument with a portable mass that can still provide enough low- $Z$ cross section to thermalize a $14-\mathrm{MeV}$ neutron is gained. The downfall of the single position-sensitive sensor in the long counter design is its low intrinsic efficiency and crude energy resolution, even in advanced designs $[5,6]$. While adding axial sensors radially can theoretically improve the intrinsic efficiency as well as the energy and spatial resolution of a long-counter, a large fraction of the moderating medium must be displaced, increasing both the volume and overall instrument mass [7]. What is needed is a different type of thermal neutron sensor that yields minimal displacement of the moderating medium, while simultaneously providing volumetric resolution of the average neutron thermalization along three-coordinate axes within the moderator-detector assembly (see Figure 1). Three-dimensional resolve is important in improving the source identification accuracy (and in increasing the number of detector responses one can unfold against for absolute neutron energy determination), while also providing the ability to achieve directional resolution. The efficacy of all the 
analysis methods described here is based on the uniqueness (between various neutron source types) of the measured intensity as a function of three-dimensional position in the moderating medium (vide infra). Based on mean free path in common moderating media, the volumetric resolution for thermal and epithermal incident and scattered neutrons should be no larger than $1 \mathrm{~cm}^{3}$, which forms the most restrictive design constraint. While state-of-the-art gaseous, doped-plastic, doped-glass, and dopedliquid thermal neutron detectors may be capable of meeting a small volume, their thermal neutron detection efficiency density is a factor of at least five times lower than advanced semiconductor-based detectors, especially when the readout electronics are also considered [8]. It is for these reasons, in addition to technological maturity, gamma-ray insensitivity, and availability, that the microstructured neutron detectors (MSNDs) [3] have been chosen for use in this work.

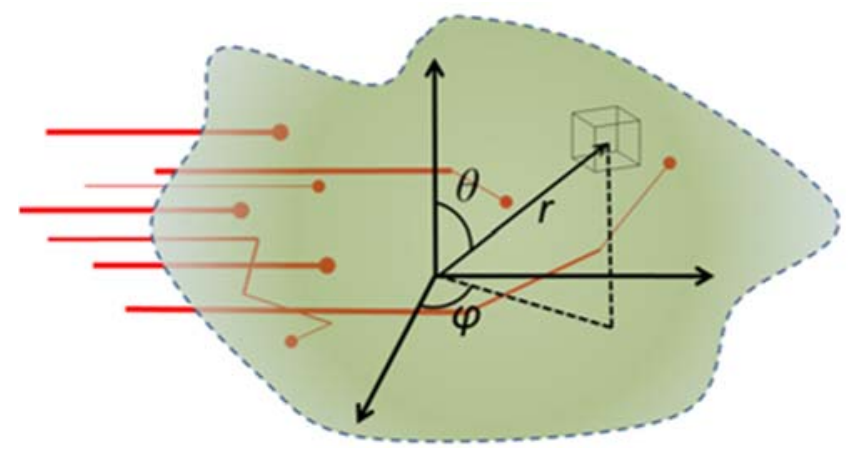

Figure 1. Illustration of free neutrons thermalizing in a moderating medium, emphasizing the concept of volumetric resolution to better than $1 \mathrm{~cm}^{3}$ along three coordinate axes (TOP). Illustration of a cylindrical moderator, and cylindrical coordinate system (BOTTOM).

\subsection{Theory of Operation}

85 Passively searching for and/or identifying neutron sources in non-proliferation applications requires the collection and analysis of one or more free neutron properties with high efficiency and energy sensitivity. To measure the neutron dose equivalent for health physics applications, identify one or more neutron sources, or determine the relative direction of a neutron source, analysis of the free neutron signature is one of the most powerful means. A high intrinsic and absolute neutron detection efficiency is important given the limited time available for measurement in these operational environments and energy sensitivity is crucial to neutron source identification and measuring the neutron dose equivalent 
94 Although spectral deconvolution (unfolding) can be used to determine absolute neutron energy, relative or proportional signatures can provide as much or more information by template matching (measuring the degree of correlation of) the calibrated intensity profile to an absolute incident neutron energy signature (i.e., thermalized neutron intensity as a function of one or more axes in a moderating medium). Further, analysis of proportional signatures does not carry the computational overhead or assumptions required in solving the classically underdetermined and ill-conditioned inverse problem $[10,11]$. To measure quantities proportional to free neutron kinetic energy using the moderating mechanism, it is critical to design an instrument configuration whose sensitivity between incident spectral features and unique thermalization is maximized. To accomplish this connection, a combination of tuned scattering and absorption materials with thermal neutron detectors is required, and is discussed in detail below.

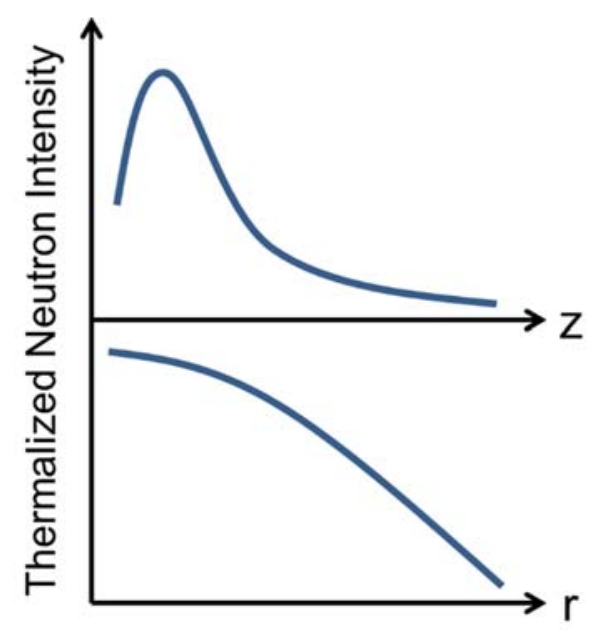

Figure 2. A heuristic illustration of the thermalized neutron intensity distribution as a function of the axial and radial position in a cylindrical moderator in cylindrical coordinates. Neutrons with a Watt energy spectrum are assumed to be incident on the front face of a cylindrical moderator $(z=0$ is the front face) and uniformly distributed over $r$.

Figure 2 is a starting point in understanding how the thermalized neutron intensity can be used as a signature of the incident neutron spectrum or the neutron source's location relative to the instrument. It is the spectral fingerprint (intensity as a function of one or more coordinate axes) uniqueness and intrinsic efficiency values, that serve as metrics for the process by which the instruments reported here are iteratively designed. Absolute neutron detection efficiency, which is determined by the area onto which the neutrons impinge, is not an essential feedback criteria of the designs considered here; rather, energy-dependent intrinsic neutron detection efficiency, that normalizes area, is used. In this work, an instrument's intrinsic neutron detection efficiency to a particular neutron source spectrum, $S$, (e.g., bare ${ }^{252} \mathrm{Cf}$, AmBe moderated by 1 in. of HDPE, etc.) is defined as 


$$
\eta_{\text {int }}(S)=\frac{N_{\text {det }}(S)}{N_{\text {inc }}(S)}
$$

where $N_{\text {det }}(S)$ is the total number of neutrons detected by the instrument from source $S$ and $N_{\text {inc }}(S)$ is the total number of neutrons from source $S$ that impinge upon the instrument volume. Figure 2 illustrates that a Maxwellian-type neutron energy spectrum will cause a reproduction of that curve shape as a function of the axial position into the moderator, and that neutron scatter and escape will cause a drop-off in intensity as a function of radius. In this simplified example, the axial and radial distributions yield a signature of the neutron source that can be compared against reference intensity distributions. The problem is then how to design the detector-moderator assembly to best resolve these signatures and/or use the captured data to generate more complex distributions for comparison using source spectra of interest to the application.

\subsection{Detector and Moderator Design}

Based on the need to detect thermalized neutrons along three-coordinate axes in $\leq 1-\mathrm{cm}^{3}$ voxels in a moderating medium with axial symmetry (e.g., Figure 1 BOTTOM), the instrument design initially converged on a 2-D array of pixelated detectors (Figure 3 LEFT) that could be stacked axially to yield a 3$\mathrm{D}$ array (Figure 3 RIGHT). With each detector read out individually, thermalized neutron intensity can be summed for any coordinate, product of coordinates, or approximate volume (e.g., quadrants, octants) that best fits the application needs (see Figure 4). For crude directional analysis, octant summing is useful (e.g., neutrons are incident from the forward-right-up position), and for crude source identification, summing each wafer plane and plotting the intensity vs. axial position can be useful (Figure 2 TOP). To enable space-filling and approximate radial symmetry, a hexagonal pattern (Figure 3 LEFT) was chosen for the first instrument design and build; later instruments used a square detector shape and square 2-D assembly to reduce the build complexity. In all, four instruments of varying mass and detector arrangement were designed, built, tested and will be discussed and compared.
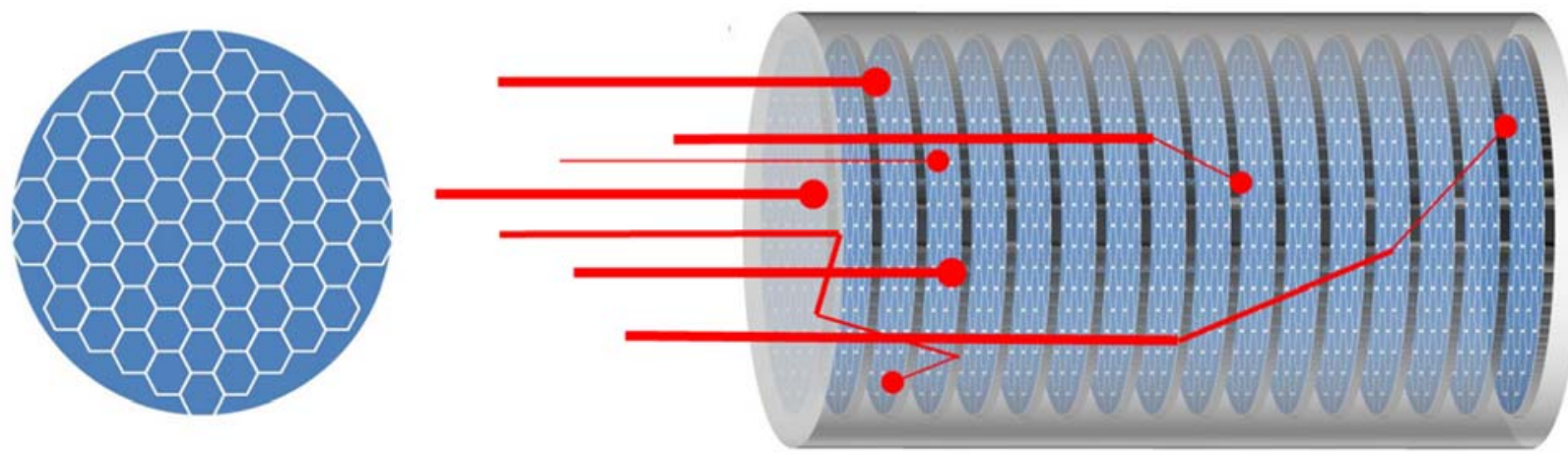

Figure 3. LEFT - illustration of a MSND board with hexagonal patterning to form a 2-D detector array with approximate radial symmetry. RIGHT - an axial stack of 2-D detector arrays, separated and embedded by neutron moderator. 

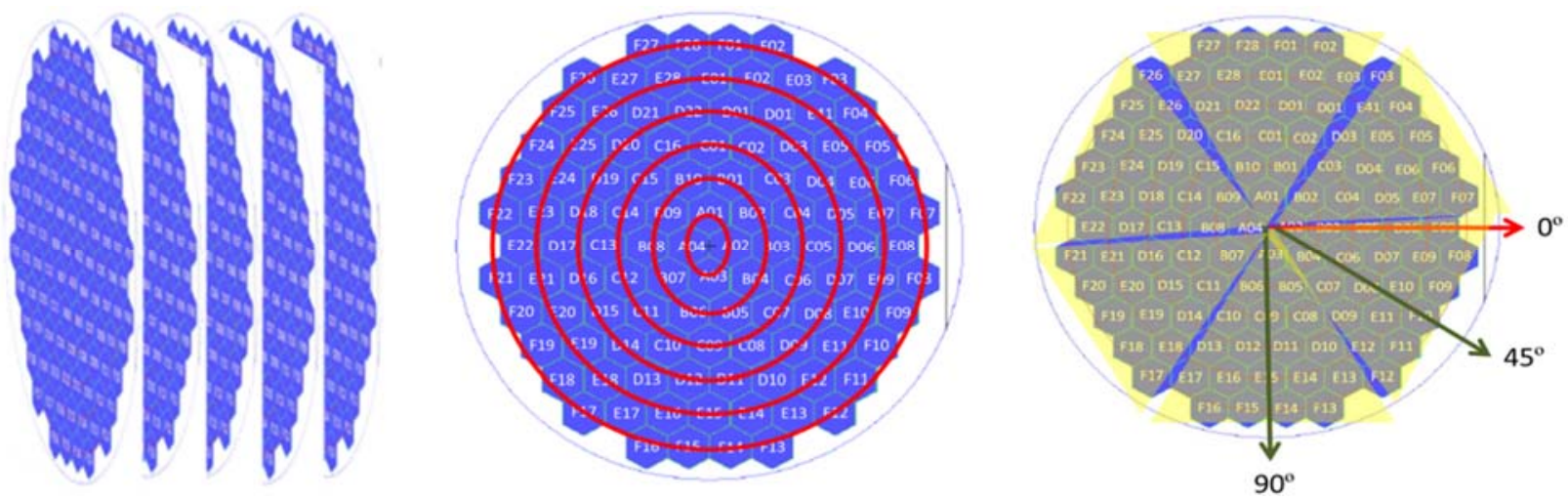

147 Figure 4. Example illustrations of detector plane intensity summations vs. axial position (LEFT), vs. radius 148 (MIDDLE), and vs. angular sector (RIGHT).

149 In addition to the individual detector shape, the 2-D detector array design requires finding the optimal 150 thermal neutron detection efficiency, active area, summed area, and summed shape(s). However, the

151

152

153

154 155

156

157

158

159

160

161

162

163

164

165

166

167 overall instrument optimization process is ultimately determined based on the intimate relationship between detector and moderator, in reference to the source spectra of interest to the application and method of operation. Therefore, the design of the detectors should be made in the context of the overall instrument, in light of its concept-of-operations and the expected spectral distribution, including environmental scattering factors.

For simplicity, the source spectra used to optimize the instrument designs described here are that of bare ${ }^{252} \mathrm{Cf}[9]$ and bare $\mathrm{AmBe}$ [9]. Although the use of other sources, including moderated variants thereof, would follow the same procedure, bare ${ }^{252} \mathrm{Cf}$ and $\mathrm{AmBe}$ were chosen due to the similarities of their induced responses in moderating-type neutron spectrometers. Operationally, the instrument can be carried or set down for measurement. Neutrons can be incident from any direction when in source search mode, however, most of the source neutrons must enter the front cylinder face to optimally perform the identification analysis while in spectral mode. It is not absolutely required that the front face represent the reference datum point, since a signature can be developed for any incident angle relative to the long-axis of a cylindrical moderator-detector assembly; the downside for non-longitudinal incident neutrons is a lower net identification certainty, the discussion of which is outside the scope of this work. It is in this case, however, that a spherical geometry could be the best theoretical design due to 3-dimensional symmetry.

The design work discussed here is based on the simultaneous optimization of: (1) the intrinsic efficiency to spontaneous fission neutron sources (spontaneous fission being the neutron source type of interest to most applications), and (2) the ability to spectroscopically differentiate spontaneous fission sources from $(\alpha, n)$ and/or spallation neutron sources (representing the ability to discern potential threats from non-threats and/or ambient background radiation), and moderated/shielded variants thereof. Using (2) as a measure of the signature sensitivity, the efficacy of varying analysis techniques for each moderatordetector design type can be compared systematically. Source differentiation is an operational feedback tool analogous to energy resolution; when the spectral fingerprint between two sources or source 
configurations is most different, the energy resolution (in the most relevant energy range) or equivalent quantity should be optimal.

To perform the signature (or energy) sensitivity analysis for proposed instrument designs, a onedimensional cross correlation analysis is used to provide an operational optimization metric. In this case, the Pearson product-moment cross-correlation coefficient [12] is used to yield a "score" between the axial intensity distributions created from a simulation of bare ${ }^{252} \mathrm{Cf}$ neutrons and a simulation of bare AmBe neutrons incident on the front face of the instrument. The Pearson correlation, $r$, (Equation 2) results in a value in the range $[-1,1]$ which is a measure of linear correlation (similarity) between the two spectrometer responses, where $r=1$ indicates a total positive correlation (the signatures are exactly the same), $r=0$ indicates no correlation (the signatures are entirely different), and $r=-1$ indicates a total negative correlation (the signatures are exactly opposite). Therefore, the energy sensitivity of a proposed instrument design is optimal when this correlation is minimized (i.e., when the two signatures are least similar and can be more easily differentiated).

$$
r_{C f, A m B e}(z)=\frac{1}{n} \cdot \sum_{i=1}^{n} \frac{N_{C f}\left(z_{i}\right)-\overline{N_{C f}(z)}}{\sigma_{N_{C f}(z)}} \cdot \frac{N_{A m B e}\left(z_{i}\right)-\overline{N_{A m B e}(z)}}{\sigma_{N_{A m B e}(z)}}
$$

where $N_{C f}(z)=\left[\begin{array}{c}N_{C f}\left(z_{1}\right) \\ \vdots \\ N_{C f}\left(z_{n}\right)\end{array}\right]$ is the ${ }^{252} \mathrm{Cf}$ response, $N_{A m B e}(z)=\left[\begin{array}{c}N_{A m B e}\left(z_{1}\right) \\ \vdots \\ N_{A m B e}\left(z_{n}\right)\end{array}\right]$ is the AmBe response,

$\overline{N(z)}$ is the average of $N(z)$, and $\sigma_{N(z)}$ is the standard deviation of $N(z)$.

In order to optimize between the maximum intrinsic efficiency and minimum Pearson correlation metrics for the second and third instrument designs, numerous simulation series were conducted using the Monte Carlo N-Particle transport code (MCNP5). For each of the simulation series described here, although most other available parameters (e.g., moderator thickness, radius, and material) are varied within the design constraints (size, weight, cost, etc.), the MCNP representation of the detector boards remains unchanged and eight detector boards were used in each simulation (unless otherwise stated). Each of the eight detector boards consists of a $4 \times 4$ square array of 2-cm $\times 2-\mathrm{cm}$ MSNDs. Each detector array is covered in front with an aluminum shield and mounted on a 10.16-cm x 10.16-cm FR-4 (E-Glass) printed circuit board (PCB) with all circuit components mounted to the side opposite the detector array (see Figure 5).

The MSNDs are comprised of Si diodes, etched to form trenches and backfilled with ${ }^{6}$ LiF powder; they yield an empirical thermal neutron detection efficiency of $\eta_{t h} \approx 22 \%$. The MSND detection mechanism has been described in detail previously [13]. To reduce computation time for the MCNP simulations described in this work, it was necessary to simplify the geometrical representation of the MSNDs. Each MSND was modeled as one $2-\mathrm{cm} \times 2-\mathrm{cm} \times 0.0525-\mathrm{cm}$ rectangular prism (true MSND outer dimensions) containing both ${ }^{6} \mathrm{LiF}$ and $\mathrm{Si}$, and the appropriate ratio of ${ }^{6} \mathrm{LiF}$ to $\mathrm{Si}$ was determined by matching the simulated and empirically measured thermal neutron detection efficiencies. To accomplish this, several preliminary simulations were run in which thermal neutrons (approximated by a Maxwellian distribution 
with a temperature parameter of $0.0257 \mathrm{eV}$ ), emitted uniformly from a planar source, were directed toward the MSND model. Since the neutron detection transduction mechanism of the MSNDs depends upon the reaction ${ }_{3}^{6} L i+{ }_{0}^{1} n \rightarrow{ }_{2}^{4} \alpha+{ }_{1}^{3} t$, the number of $(n, t)$ reactions (i.e., neutron capture reactions in which tritons, ${ }_{1}^{3} \mathrm{H}$ nuclei, are produced) occurring in the lumped MSNDs were tallied (counted) in each simulation, and the thermal neutron detection efficiency, $\eta_{t h}$, was calculated by

$$
\eta_{t h}=\frac{N_{(n, t)}}{N_{t h}}
$$

where $N_{(n, t)}$ is the total number of $(n, t)$ reactions occurring in the lumped MSNDs and $N_{t h}$ is the total number of thermal neutrons that impinge upon the Lumped MSNDs. Keeping the percentages of ${ }^{6} \mathrm{Li}$ and $\mathrm{F}$ equal, the ratio of ${ }^{6} \mathrm{LiF}$ to $\mathrm{Si}$ was adjusted in each simulation until $22 \%$ thermal neutron detection efficiency was achieved.

\subsubsection{Moderator Geometry and Dimension Optimization}

Although one could consider many other geometries, only the cylindrical and rectangular prism moderator geometries were designed and built (Figure 5) using high-density polyethylene (HDPE). A spherical design offers the most flexibility and can intrinsically mitigate the effect of environmentscattered or multi-directional neutrons, and conical designs may show merit, but their manufacturing complexity and/or required thermalization mass is too high. Figure 6 shows the simulated intrinsic neutron detection efficiency and spectral sensitivity for both the cylindrical and rectangular prism designs as a function of moderator radius, $R$, and thickness, $T$, between each of the eight detector boards. In the case of the rectangular prism, the radius is defined, as shown in figure 5 , by the distance from the center to the corner of the moderator's front face.
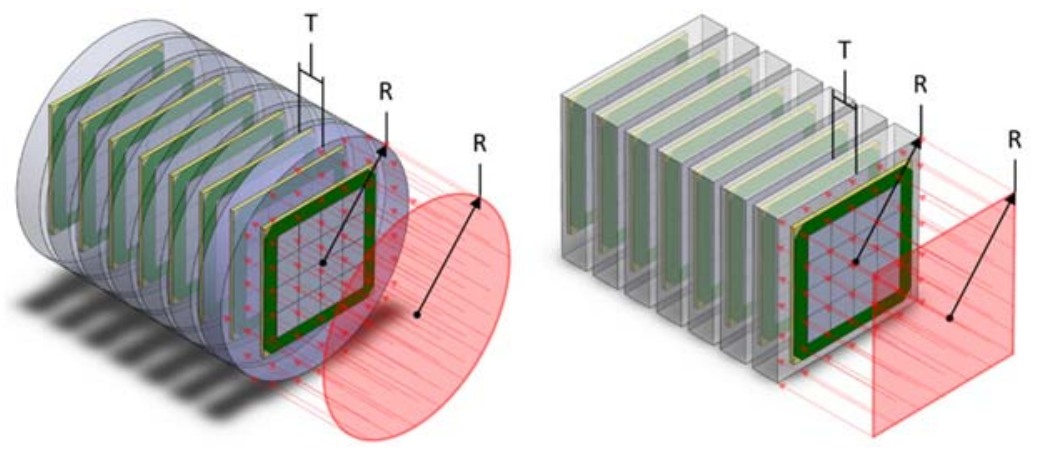

Figure 5. Renderings of the cylindrical (LEFT) and rectangular prism (RIGHT) moderator geometries with eight $4 \times 4$ arrays of $4-\mathrm{cm}^{2}$ detectors, and the thickness and radius of the moderator slabs indicated. The red plane represents the neutron source used in the simulations to produce the results in Figure 6. 

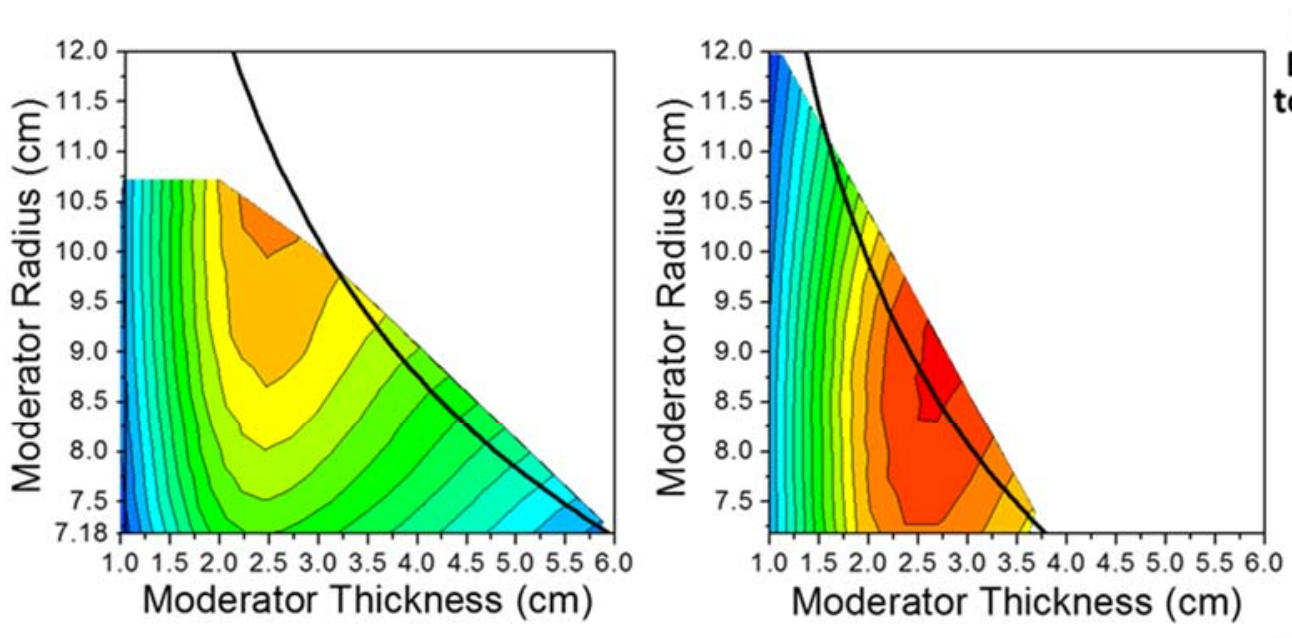

Intrinsic

Efficiency

to ${ }^{252} \mathrm{Cf}(\%)$
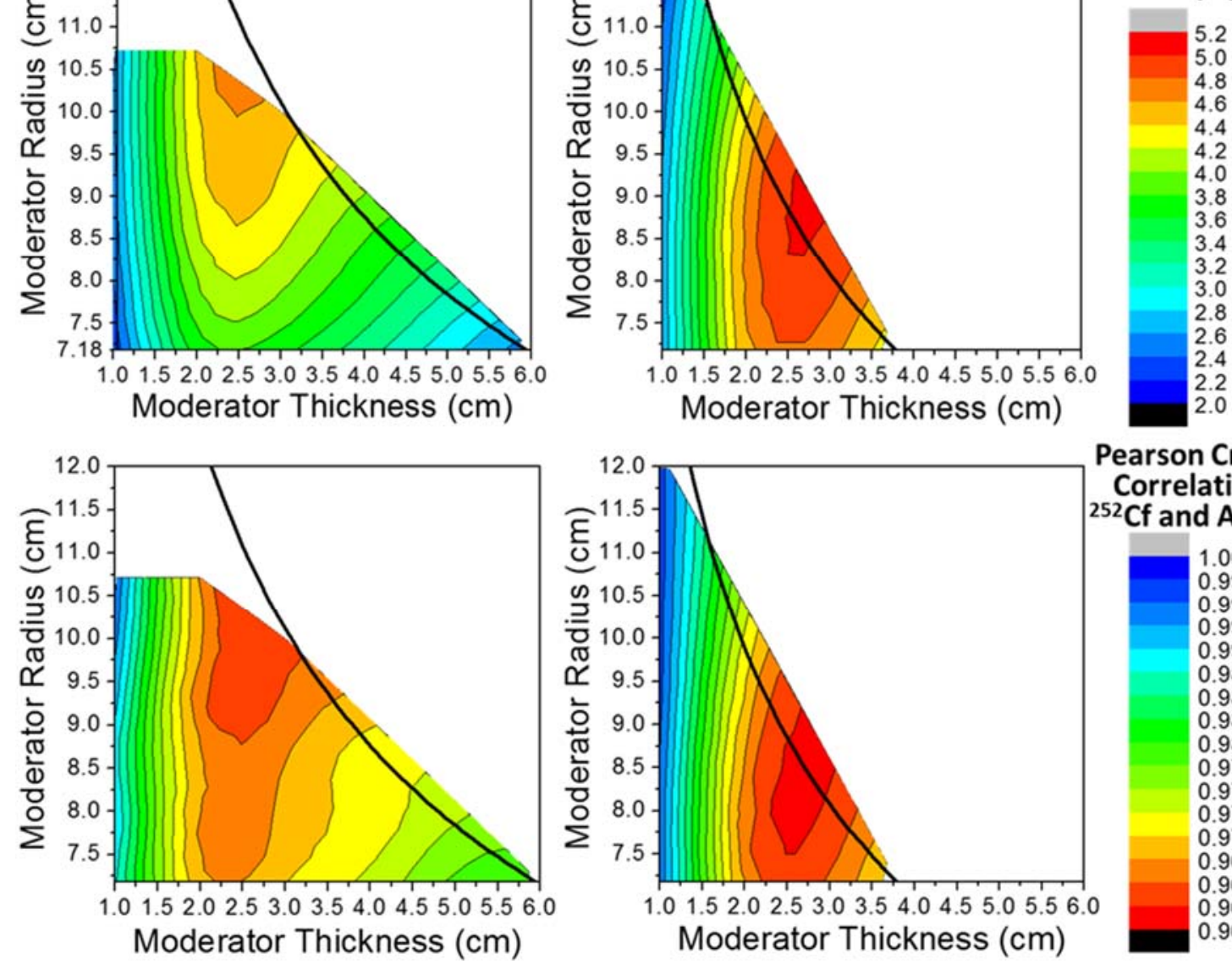

Pearson CrossCorrelation: ${ }^{252} \mathrm{Cf}$ and $\mathrm{AmBe}$

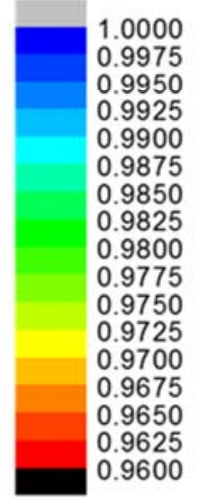

Figure 6. Results of the simulated intrinsic neutron detection efficiency to bare ${ }^{252} \mathrm{Cf}$ for the rectangular prism (TOP LEFT) and cylindrical (TOP RIGHT) geometries, with a common color scale (red indicating maximum intrinsic efficiency). And results of the simulated spectral sensitivity metric $\left({ }^{252} \mathrm{Cf}-\mathrm{AmBe}\right.$ spectral difference) for the rectangular prism (BOTTOM LEFT) and cylindrical (BOTTOM RIGHT) geometries, with a common color scale (red indicating minimum Pearson correlation). The thick black line in each plot is an iso-mass curve, indicating the radius and thickness combinations that yield a total moderator + detector board weight of $10 \mathrm{lbs}$.

The instrument optimization and analysis methods used to create Figure 6 serve as a primary theoretical baseline and operational feedback tool for the instrument designs described in this work. As stated previously, the $4 \times 4$ array detector boards are considered here to be a fixed design parameter for investigating the effects of moderator geometries and dimensions on the aforementioned operational performance metrics for detection efficiency and source identification (energy sensitivity). The limit of eight detector boards per instrument is estimated based on conservatively-projected cost limitations, forming another fixed design parameter. Holding these two parameters constant, the physical moderator dimensions described in Figure 5 are varied under an active weight constraint of $10 \mathrm{lbs}$., and simulated for a course grid of discrete radius and thickness combinations (representing single $(x, y)$

251 points in each plot in Figure 6). Intrinsic neutron detection efficiencies to bare ${ }^{252} \mathrm{Cf}$ and Pearson 
correlations between bare ${ }^{252} \mathrm{Cf}$ and bare $\mathrm{AmBe}$ instrument responses are then calculated for each discrete radius and thickness combination and plotted in a third (metric) dimension. A threedimensional interpolation is then applied to the data, indicating regions of the tunable parameter space that may warrant further exploration. These promising regions are then broken down into finer grids of discrete points for subsequent simulation, and the process is repeated until no practically significant improvement can be made toward the desired optimization metric. This optimization technique is

258 explored further and utilized to a fuller extent in following sections.

259 In analysis of Figure 6, the most immediately apparent result is that, for an active instrument weight of 260 less than $10 \mathrm{lbs}$., the cylindrical moderator geometry outperforms the rectangular prism geometry for 261 both operational metrics. Since portability is of primary concern to both nuclear nonproliferation and 262 health physics applications, the cylindrical geometry is clearly more optimal for meeting the application 263 needs under the simplifying assumptions made here. It is also significant (and serendipitous) to note 264 that, for both geometry types, the optimal moderator thickness is roughly the same for both 265 performance metrics, indicating that simultaneous optimization may be possible without significant 266 tradeoffs to either metric.

\section{2.3.2 Detector Efficiency Optimization}

268 Commercially available microstructured semiconductor neutron detectors are capable of thermal 269 neutron detection efficiencies in excess of $44 \%[14,20]$. To explore the effect of increasing thermal 270 neutron detector efficiency of the constituent MSNDs on the intrinsic neutron detection efficiency of a 271 cylindrical spectrometer design, a series of simulations was conducted in which neutrons from a bare

$272{ }^{252} \mathrm{Cf}$ source were incident upon the front circular face of a moderator-detector assembly with thirty 5-

273 inch diameter cylindrical detector boards, separated axially by 1-cm of HDPE with 5-inch diameter (initial

2745 5 design, Figure 3, discussed further in Section 3). The thermal neutron detection efficiencies of the 275 constituent MSNDs were varied in each simulation from $3 \%$ to $51 \%$. Figure 7 shows that as the thermal 276 detection efficiencies of the MSNDs increases above $22 \%$ there is a diminishing return to the instrument 277 intrinsic efficiency to bare ${ }^{252} \mathrm{Cf}$. Quantitatively, the intrinsic neutron detection efficiency more than 278 quadruples (from $0.669 \%$ to $2.85 \%$ ) when using a $22 \%$ detector over a $4 \%$ detector, while only increasing 279 by a factor of 1.68 (from $2.85 \%$ to $4.79 \%$ ) when using a $44 \%$ detector over a $22 \%$ detector. Due to this 280 diminishing return in intrinsic efficiency, the instruments designed in this work were held to $22 \%$ 281 thermal neutron detection efficiency MSNDs. 


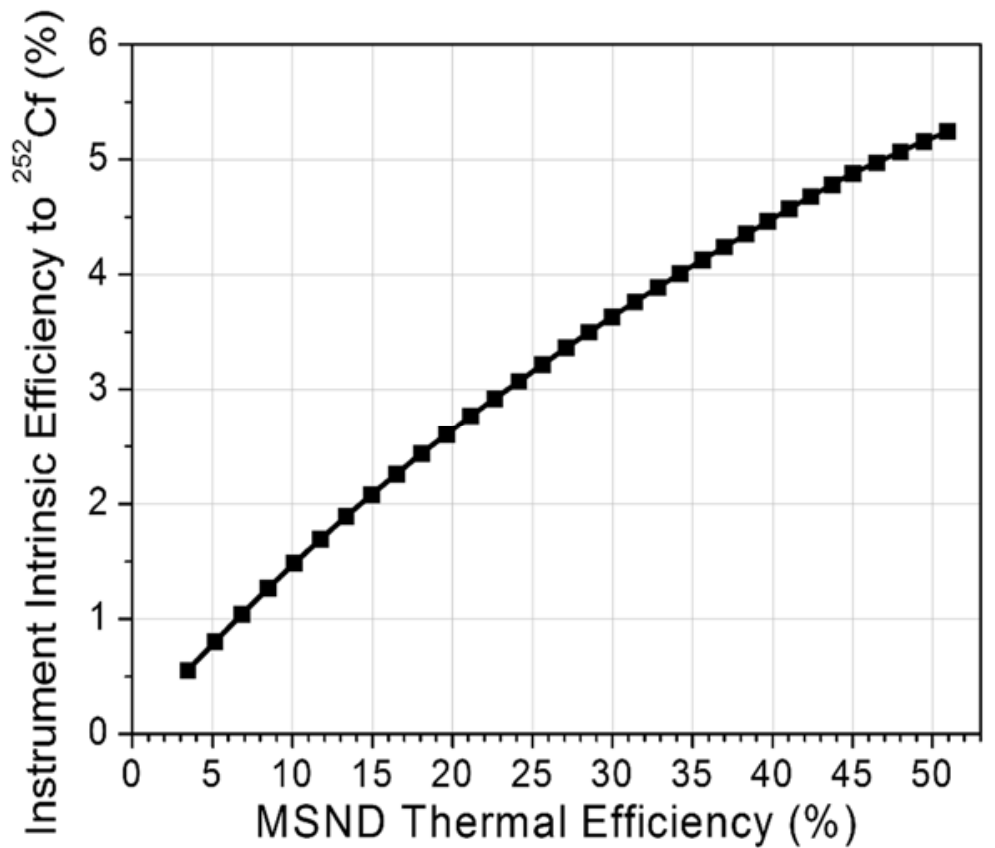

283 Figure 7. Simulated instrument intrinsic detection efficiency to ${ }^{252} \mathrm{Cf}$ as a function of MSND thermal 284 neutron detection efficiency.

\subsubsection{Moderator Radius to Detector Radius Ratio Optimization}

As shown in Figure 2 (BOTTOM), in the case that the detector plane is the same radius or area as the axially symmetric moderator, the intensity of neutron counts (from normal incident on the front cylinder face) fall off as a function of radius. If a neutron reflecting material is placed outside of the outer detector radius, some neutrons can be re-scattered or reflected inward and the intrinsic neutron detection efficiency can increase. Figure 8 shows how an instrument's intrinsic efficiency to bare ${ }^{252} \mathrm{Cf}$ changes as a function of outer detector radius for various moderator-to-detector radius ratios. In these simulations, high density polyethylene is used as both the central moderator and reflector. In all simulations with an outer detector radius less than $5 \mathrm{~cm}$, the intrinsic efficiency increases as the moderator-to-detector radius ratio increases. Above a $5-\mathrm{cm}$ outer detector radius the intrinsic efficiency increase slows and reaches a maximum of $36 \%$ at a $10-\mathrm{cm}$ outer detector radius for the 1.125 and 1.25 moderator-to-detector radius ratios. The falloff above a $10-\mathrm{cm}$ outer detector radius for all moderatorto-detector ratios is due to the moderator radius being larger than the average mean free path of the bare ${ }^{252} \mathrm{Cf}$ neutrons in high density polyethylene. This leads to increased neutron absorption by hydrogen nuclei in the moderator volume $\left({ }_{0}^{1} n+{ }_{1}^{1} \mathrm{H} \rightarrow{ }_{1}^{2} \mathrm{H}+\gamma(2.223 \mathrm{MeV})\right)$. For a portable instrument with cylindrical symmetry and total weight of $\sim 15$-lbs., the moderator volume, at maximum, would be restricted to a $7.6-\mathrm{cm}$ radius and $30-\mathrm{cm}$ length. To achieve the highest intrinsic efficiency (not necessarily the highest absolute efficiency) for the cylindrical geometry ( 15\%) and total weight of 15Ibs., the detector radius would not exceed $3.8-\mathrm{cm}$. 


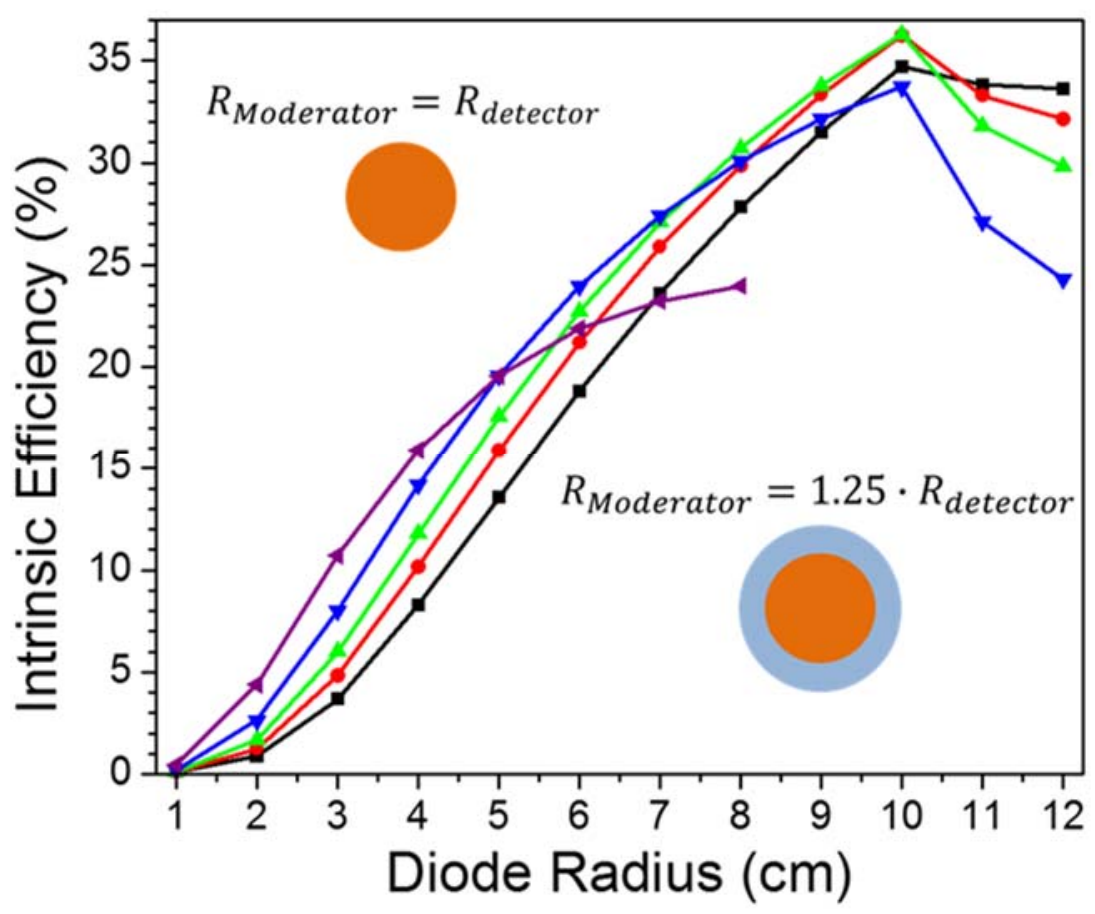

305 Figure 8. Intrinsic neutron detection efficiency to ${ }^{252} \mathrm{Cf}$ as a function of the detector (diode) radius for varying detector to moderator radii ratios ( $\square R_{\text {moderator }}=R_{\text {detector }}, \bullet R_{\text {moderator }}=1.125 \cdot R_{\text {detector }}$, $\Delta R_{\text {moderator }}=1.25 \cdot R_{\text {detector }}, \nabla R_{\text {moderator }}=1.5 \cdot R_{\text {detector }},\left\langle R_{\text {moderator }}=2 \cdot R_{\text {detector }}\right)$.

\subsubsection{Moderator Type Optimization}

309 A major goal of this work is to design an instrument that will yield the most unique distribution of detected thermalized neutrons while also maintaining the highest possible intrinsic efficiency. Since the type of neutron moderating material used can greatly influence both of these operational characteristics, it is important to consider the role of moderators other than high density polyethylene (HDPE). HDPE is a popular neutron moderating material because it is inexpensive, readily available, mechanically strong but easily machinable, and resilient to most environmental factors. Its hydrogen concentration by volume is very high (i.e., $8.07702 \mathrm{E}+22$ Atoms $\mathrm{H} / \mathrm{cm}^{3}$ ), which is important for neutron scattering and moderation. However, could a very high hydrogen concentration also be detrimental to thermal and lower-epithermal energy resolution, as neutrons in these energy ranges can be thermalized and detected with little traverse into the instrument detector layers? Or conversely, could a higher hydrogen concentration material, or possibly high carbon containing, help to improve the thermalized intensity distribution (i.e., signature of higher-epithermal or fast neutrons) by better physical separation of the higher energy neutrons detected? 


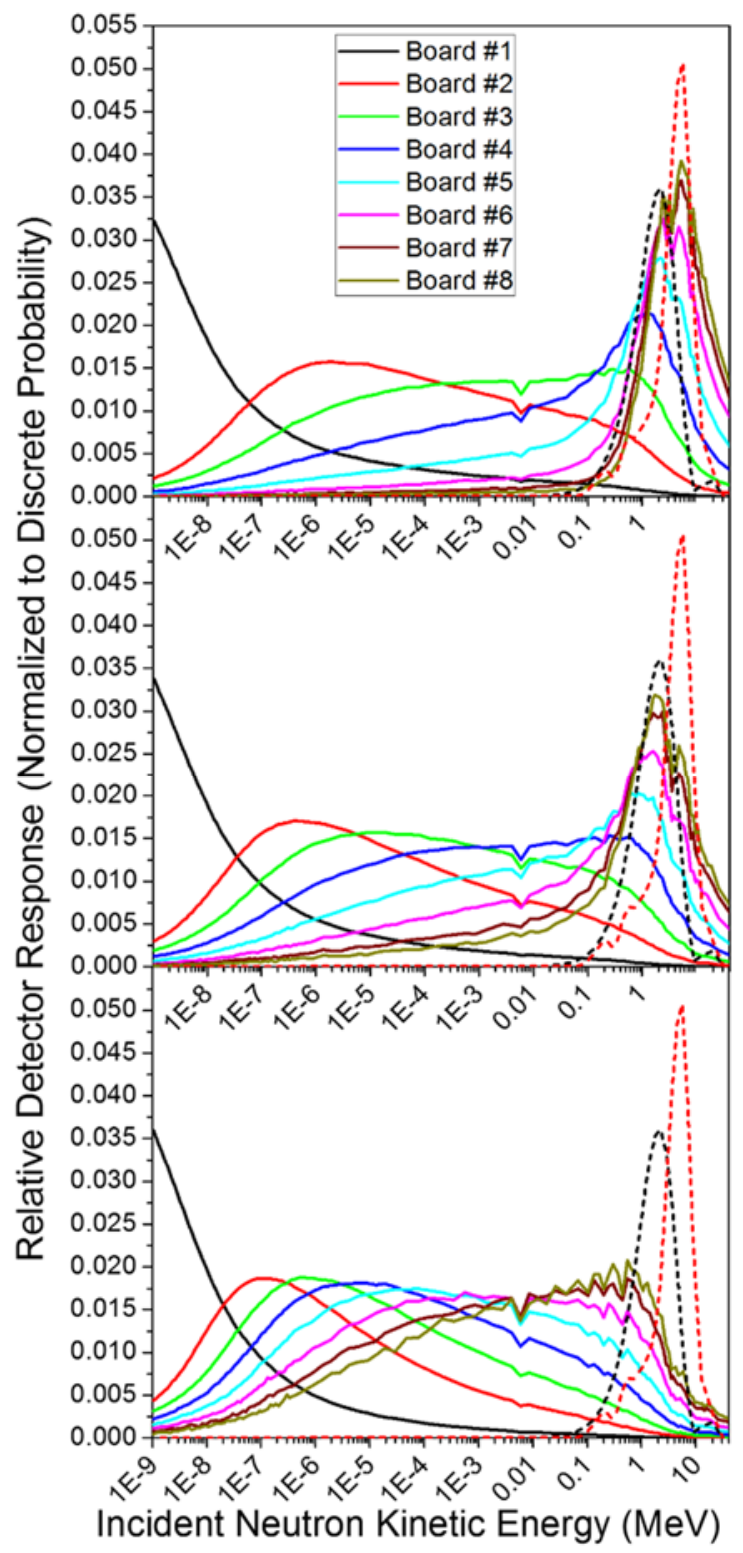

323 Figure 9. Shown (ALL) are normalized detector-board-summed response functions (solid lines) of instruments with a cylindrical moderator design with $7.62-\mathrm{cm}$ radius and $3.6-\mathrm{cm}$ thickness, as defined by

325 Figure 5, with 8 rectangular detector boards. Simulated moderator materials were varied with HDPE

326 (TOP), polystyrene (MIDDLE), and polysulfone (BOTTOM). In each case, the first detector board (most sensitive to the lowest energy neutrons) is represented in black and is leftmost, while the last detector

328 board (most sensitive to the highest energy neutrons) is represented by olive and is rightmost.

329 Normalized ${ }^{252} \mathrm{Cf}(---)$ and $\mathrm{AmBe}(---)$ neutron emission spectra are also included in each plot for 330 reference.

331 To help answer these questions, simulations were performed using high-density polyethylene (HDPE), 332 polystyrene, and polysulfone moderator materials, all in the same instrument configurations, in order to 333 study the effect of varying hydrogen and carbon concentration on the overall performance of the 
instrument; a summary of each moderator's salient properties is shown in Table 1. Figure 9 shows how the detector response functions vary for HDPE, polystyrene, and polysulfone in one of the tested instrument configurations $(7.62-\mathrm{cm}$ radius and $3.6-\mathrm{cm}$ thickness, as defined in Figure 5 LEFT). Figures 10 and 11 were constructed using the optimization procedures described in section 2.3.1; however, the weight restriction of the initial data point grid was relaxed and the radius-thickness parameter space was explored more thoroughly. Figure 10 depicts the effect that the varied moderator type had on the instrument's intrinsic detection efficiency to bare ${ }^{252} \mathrm{Cf}$. Figure 11 shows how the spectral sensitivity metric (Pearson cross-correlation between bare ${ }^{252} \mathrm{Cf}$ and bare $\mathrm{AmBe}$ instrument responses, Equation 2) is affected by the different moderators.

\begin{tabular}{|c|c|c|c|}
\hline Moderator & Chemical Formula & H-Conc. (Atoms/cm $\left.{ }^{3}\right)$ & C-Conc. (Atoms/cm ${ }^{3}$ ) \\
\hline High-Density Polyethylene & $\left(\mathrm{C}_{2} \mathrm{H}_{4}\right)_{\mathrm{n}}$ & $8.07702 \mathrm{E}+22$ & $4.29172 \mathrm{E}+22$ \\
\hline Polystyrene & $\left(\mathrm{C}_{8} \mathrm{H}_{8}\right)_{\mathrm{n}}$ & $4.85521 \mathrm{E}+22$ & $4.85521 \mathrm{E}+22$ \\
\hline Polysulfone & $\left(\mathrm{OC}_{6} \mathrm{H}_{4} \mathrm{OC}_{6} \mathrm{H}_{4} \mathrm{SO}_{2} \mathrm{C}_{6} \mathrm{H}_{4}\right)_{\mathrm{n}}$ & $2.76176 \mathrm{E}+22$ & $4.14264 \mathrm{E}+22$ \\
\hline
\end{tabular}

Table 1. Potential instrument moderator material salient properties.

The response functions' peak positions and full width at half maximums (FWHM) provide another means by which spectral sensitivity can be analyzed [15]. As an example, if all of the response functions were non-overlapping delta functions, the energy resolution would be perfect at each response's energy but the sensitivity would be limited only to those energies and, hence, the efficiency would be very small and unacceptable. On the other side, if all of the response functions span the entire energy range, and have the same shape and relative efficiency, the system yields no energy resolution, which is also unacceptable. The desired response functions are those that are narrow in energy, numerous, next to each other with little overlap, and cover the energy range of interest to the application. 


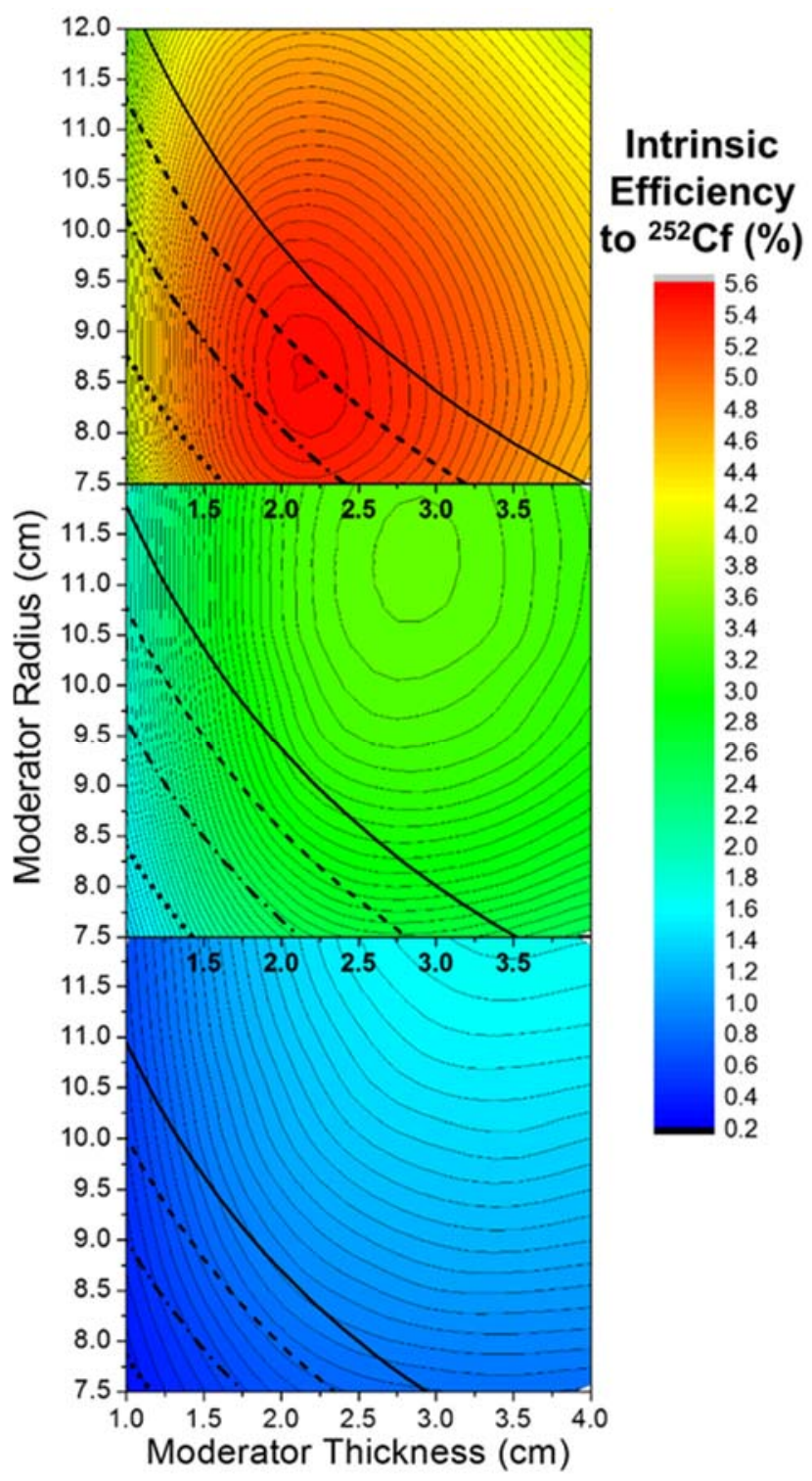

353 Figure 10. Instrument intrinsic efficiency to ${ }^{252} \mathrm{Cf}$ as a function of moderator radius and thickness using 354 HDPE (TOP), polystyrene (MIDDLE), and polysulfone (BOTTOM), with iso-mass curves indicating the 355 radius and thickness combinations that yield a total moderator + detector board weight of 6-lbs. ( $\cdots)$, 8356 Ibs. $(-\cdot-\cdot), 10-$ lbs. $(---)$, and 12-lbs. (-).

357 As shown in Figure 9, as the atomic ratio of hydrogen to carbon is decreased from $\mathrm{H} / \mathrm{C}=2.0$ (HDPE) to $358 \mathrm{H} / \mathrm{C}=1.0$ (polycarbonate) to $\mathrm{H} / \mathrm{C}=0 . \overline{6}$ (polysulfone), the peak position of each detector board response 359 function (with the exception of the front board which has no anterior moderator) is shifted to a lower 360 energy. While this shift to lower energies provides more even coverage of the entire $10^{-9} \mathrm{MeV}$ to 40 $361 \mathrm{MeV}$ energy range shown in Figure 9, the vast majority of neutron emissions from bare ${ }^{252} \mathrm{Cf}$ and $\mathrm{AmBe}$ 362 (dashed black and red lines in Figure 9 respectively) exceed $10^{-1} \mathrm{MeV}$. The effects of the low energy shift 363 on spectrometer performance are evident from Figures 10 and 11, which depict a significant decrease in 364 instrument intrinsic efficiency to bare ${ }^{252} \mathrm{Cf}$ and increase in Pearson correlation between bare ${ }^{252} \mathrm{Cf}$ and 
AmBe instrument responses with decreasing hydrogen-to-carbon atomic ratio. However, while these trends are detrimental to instrument performance in application to bare spontaneous fission and $(\alpha, n)$ neutron sources, they may be beneficial to performance in application to moderated/shielded variants thereof. After evaluating the effect of this low energy shift on moderated source types, it was determined that the performance benefits for moderated neutron sources are far outweighed by the detriment to bare neutron source performance, and HDPE proved to be the most optimal for a wider range of relevant applications.

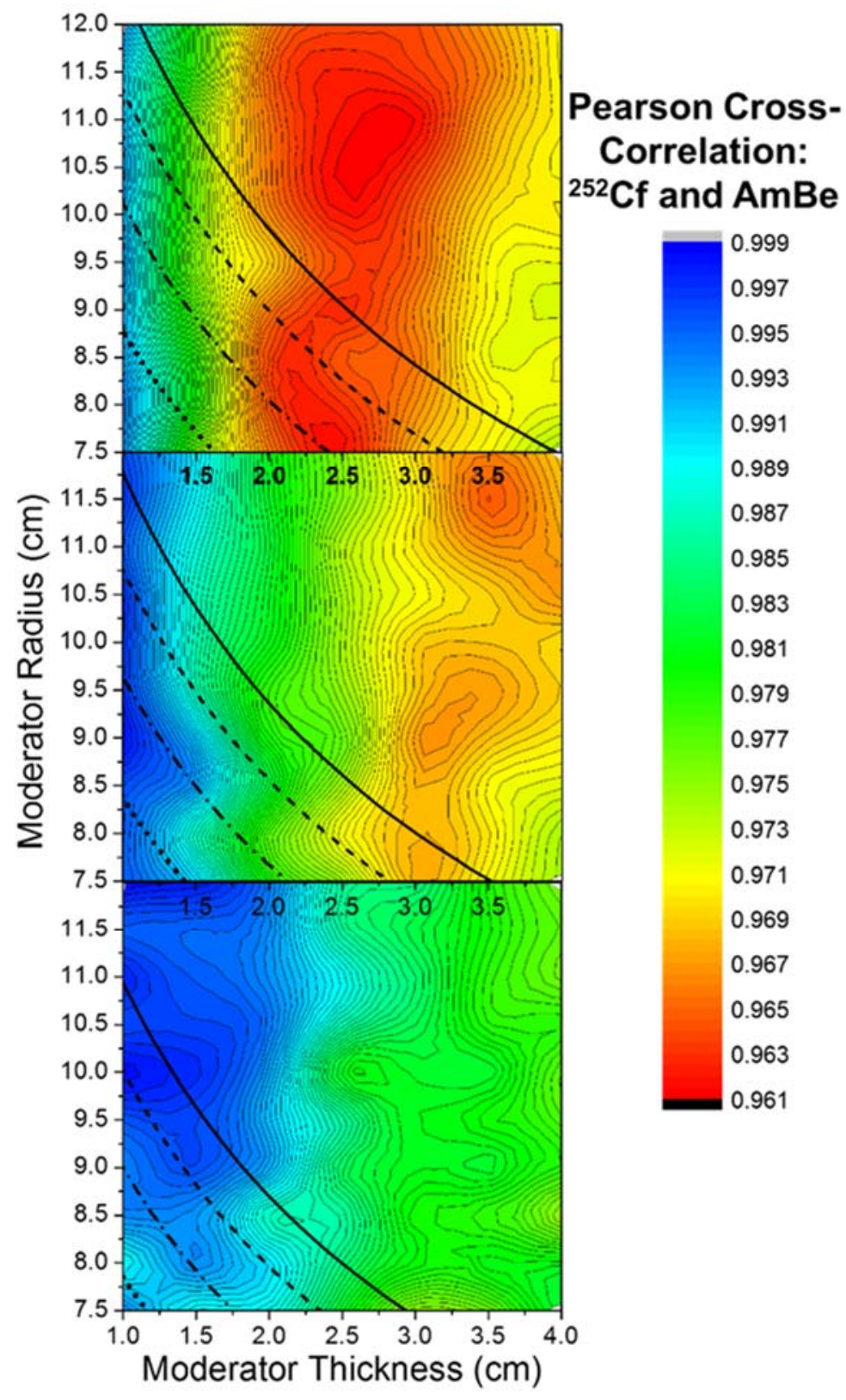

373 Figure 11. Pearson Cross-Correlation between ${ }^{252} \mathrm{Cf}$ and $\mathrm{AmBe}$ instrument responses as a function of 374 moderator radius and thickness using HDPE (TOP), polystyrene (MIDDLE), and polysulfone (BOTTOM), 
with iso-mass curves indicating the radius and thickness combinations that yield a total moderator + detector board weight of 6 -lbs. ( $\cdots), 8$-lbs. $(-\cdot-\cdot), 10$-lbs. $(---)$, and 12 -lbs. $(-)$.

\subsubsection{The Role of Absorbers}

Just as varying the moderator type can change the macroscopic neutron scattering distribution, and hence alter the response, intrinsic efficiency, and spectral sensitivity, so too can neutron absorbing materials. As shown in prior work for the long counter and similar geometries to those considered here [16-19, 21, 22], the addition of absorbers can provide two important physical attributes. First, if the neutron absorbers are tuned to capture neutrons at an energy threshold and/or resonance, and those thresholds cover many decades of energy and are strategically placed in space (e.g., stacked), they can act to filter neutrons by energy; this trait alone (i.e., without moderator) creates a crude neutron spectrometer, and can enhance a moderating-type neutron spectrometer. Second, neutron absorbers placed behind stacked detectors, in a moderating-type neutron spectrometer, act to capture some backscattered neutrons. Most backscattered neutrons cause a loss of energy resolution as they defeat the axial depth dependence of forward down-scattering. However, absorbers simultaneously decrease the intrinsic neutron detection efficiency of the instrument and usually cause secondary reaction products (e.g., capture gamma-rays) so their use should be carefully considered in light of the gammaray blindness or discrimination capability of the neutron detectors. In the case of the instruments described here, neutron absorbers were serendipitous and unavoidable, as the semiconductor-based neutron detectors were mounted to $0.81-\mathrm{mm}(0.032$ ") thick FR4 printed circuit board (PCB), containing ${ }^{79} \mathrm{Br},{ }^{81} \mathrm{Br}$, and ${ }^{10} \mathrm{~B}$. The FR4 PCBs absorb $16 \%$ of the thermalized neutrons at each detector layer, improving energy resolution at the cost of decreased intrinsic neutron detection efficiency.

\subsection{Instrument Results}

Based on the design methods described above, four moderating-type neutron spectrometers were built to test and compare the empirical response of different instrument configurations. Here forward, the instrument systems will be referred to as the 5 -inch cylinder $(\mathbf{5 C})$, shown in Figure 12, the 6-inch cylinder (6C), shown in Figure 13, the 4-inch rectangular prism (4RP), shown in Figure 15, and the 4-inch cylinder (4C), shown in Figure 16. 

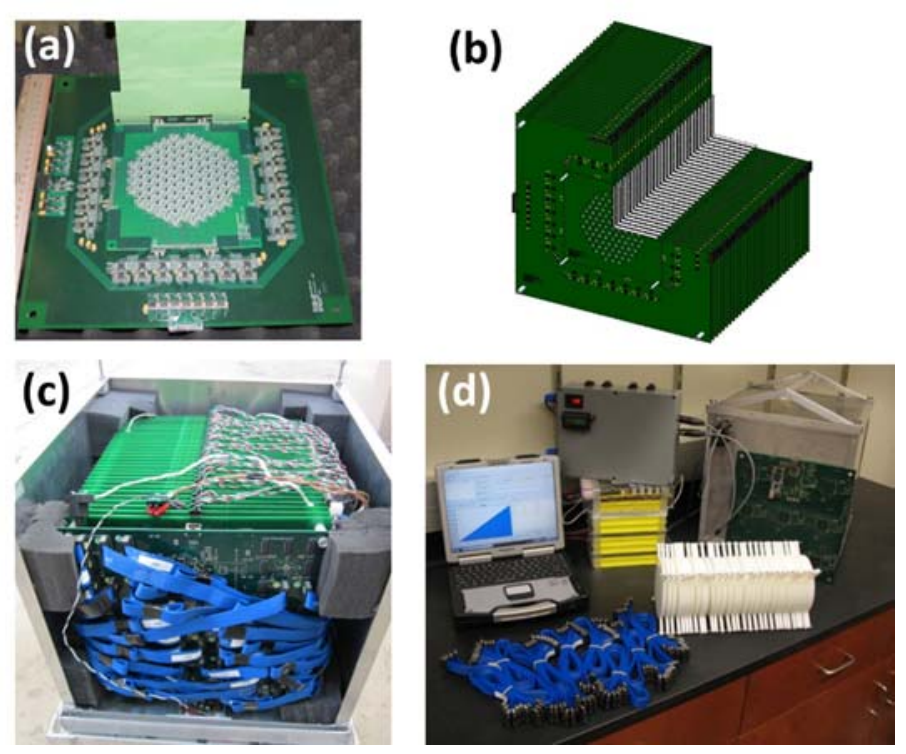

403 Figure 12. Photographs and renderings of the $\mathbf{5 C}$ system showing (a) an individual printed circuit board 404 with mounted microstructured neutron detector die, (b) assembly cut-away view showing thirty 405 alternating board-moderator pairs, (c) assembled instrument with the top and back cover removed, and 406 (d) instrument case, HDPE moderator, cables, battery, power supply, battery, and digital signal 407 processing board.

408 The $\mathbf{5 C}$ was the first instrument built; comprised of thirty detector boards, each containing 108 409 hexagonally-shaped 1- $\mathrm{cm}^{2}$ MSNDs and arranged into the pattern shown in Figure 4. On each detector 410 board, all 108 MSNDs were etched as a single monolith into a 5-inch diameter silicon wafer and read out 411 individually through coaxial cables to a digital signal processing board, containing 8 field-programmable 412 gate arrays for the 3240 total channels. When completed, the moderator and detector board assembly 413 weighed 48-lbs. without the case, battery, power supply or laptop. Due to this nearly non-portable 414 mass, overall complexity, and excessive number of channels (vide infra), the next three designs focused 415 on reducing the overall mass and volume, while improving operational reliability and effectiveness. 

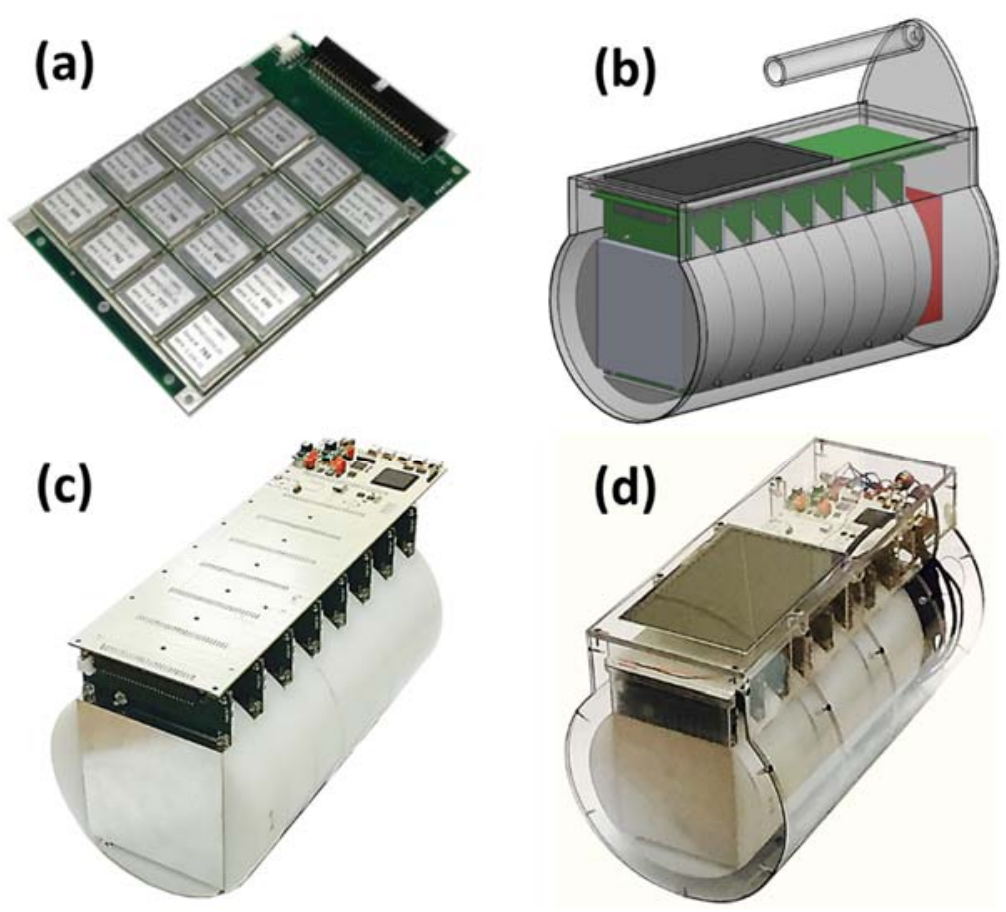

417 Figure 13. Photographs and renderings of the $6 \mathrm{C}$ system showing (a) an individual printed circuit board 418 with mounted microstructured neutron detectors, (b) assembly view showing the eight boards with 419 recessed moderator slabs, motherboard, Nexus 7 tablet, battery pack, and case (transparent), (c) 420 assembled instrument with the case, Nexus 7 tablet, and battery packs removed, and (d) fully assembled $4216 \mathrm{C}$ neutron spectrometer.

422 In the $\mathbf{6 C}$ and $\mathbf{4 R P}$ designs, cable connections were removed and the power conditioning, battery, and 423 computer were moved internal to the case. The detector daughter boards are composed of a $4 \times 4$ array 424 of $4-\mathrm{cm}^{2}$ detectors, shown in Figure 13a, with a connector that plugs directly into a motherboard above 425 the moderator volume (Figure 13c). Both the 6C and the 4RP designs contain eight daughter boards for 426 a total of 128 individual detector channels per instrument, each channel being read into a single Xilinx 427 Spartan 3AN FPGA. A Nexus 7 tablet serves as the user display, information processor, and control 428 center of the motherboard. 


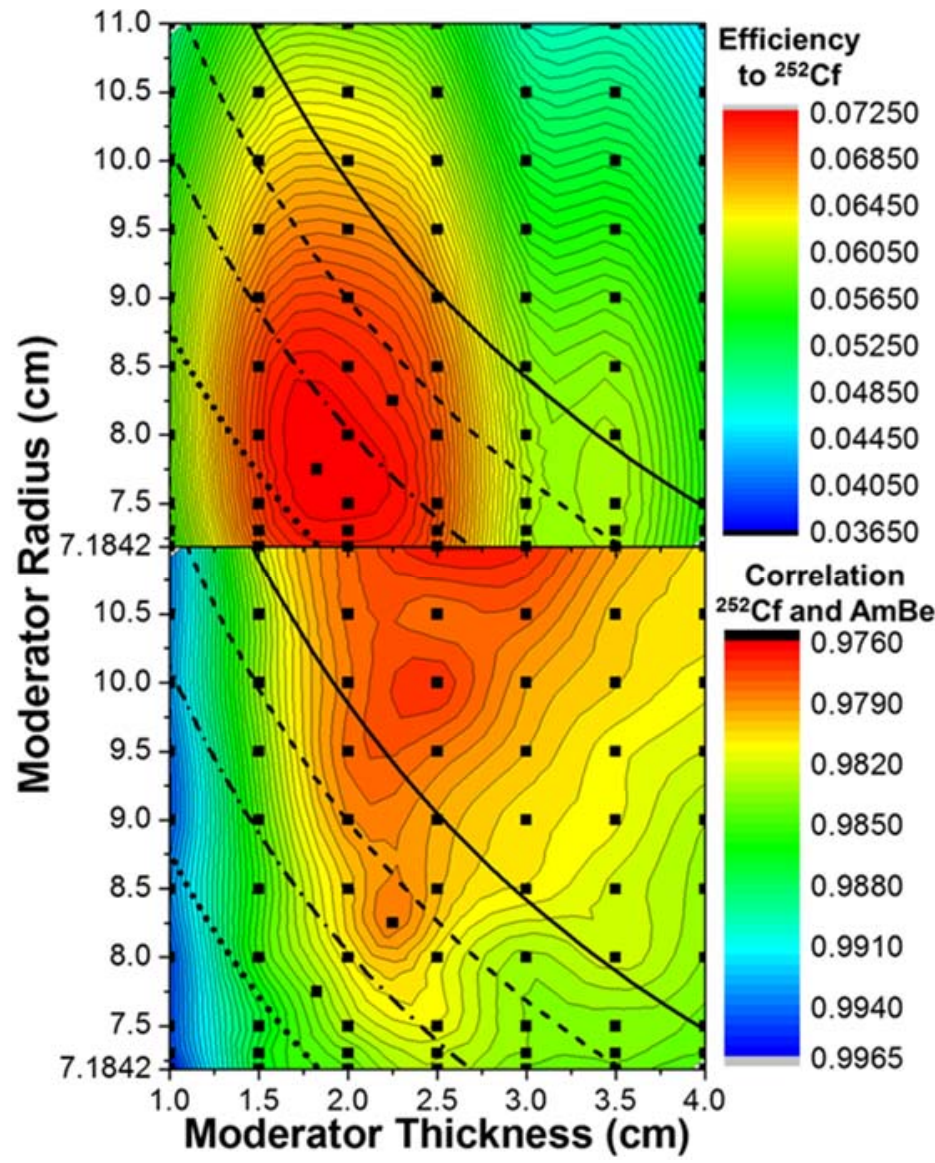

430 Figure 14. 6C spectrometer intrinsic efficiency to ${ }^{252} \mathrm{Cf}$ (TOP) and Pearson Cross-Correlation between $431 \quad{ }^{252} \mathrm{Cf}$ and $\mathrm{AmBe} 6 \mathrm{C}$ spectrometer responses (BOTTOM) as a function of HDPE moderator radius and 432 thickness, with iso-mass curves indicating the radius and thickness combinations that yield a total 433 moderator + detector board weight of 6 -lbs. ( ..), 8-lbs. (- - -), 10-lbs. (- - -), and 12-lbs. (-). • represent initially simulated data points, as well as optimal data points for each metric (<10 lbs.).

435 Using the optimization procedures described in Section 2.3.1, and restricting the total weight of the moderator and detector boards to be less than $10 \mathrm{lbs}$. as a design constraint, the optimal thickness and radius of the $6 \mathrm{C}$ moderator slabs (as defined in Figure 5 LEFT) was found to be $2.29 \mathrm{~cm}$ and $7.62 \mathrm{~cm}$ 438 respectively, as represented in Figure 14. This radius and thickness combination is a compromise between the optimal intrinsic detection efficiency, at $T=1.825 \mathrm{~cm}$ and $R=7.75 \mathrm{~cm}$, the optimal spectral 440 difference, at $T=2.25 \mathrm{~cm}$ and $\mathrm{R}=8.25 \mathrm{~cm}$, and practical availability, with more emphasis given to the 441 intrinsic detection efficiency metric. It should be noted that the $\mathbf{6 C}$ moderator slabs envelope the 442 detectors, not just separate them (as portrayed in Figure 5), which leads to a significant decrease in 443 neutron leakage, despite the small increase in mass. Therefore, with the detector board recess 444 accounted for, the total moderator slab thickness used was $3.6 \mathrm{~cm}(1.31-\mathrm{cm}$ deep detector board recess, $4452.29 \mathrm{~cm}$ between detector boards, as shown in Figure $13 \mathrm{~b}$ and $13 \mathrm{c}$ ). 

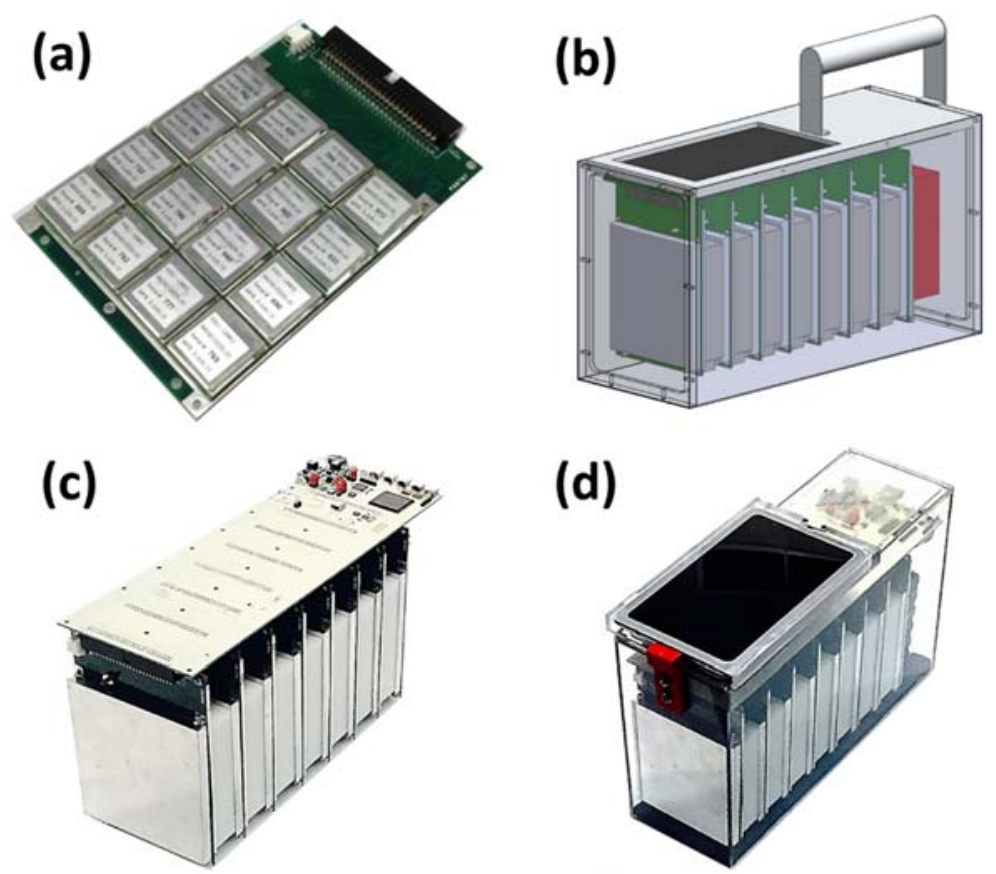

447 Figure 15. Photographs and renderings of the 4RP system showing (a) an individual printed circuit board 448 with mounted microstructured neutron detector die, (b) assembly view showing the eight alternating 449 detector-moderator pairs, motherboard, Nexus 7 tablet, battery pack, and case (transparent), (c) 450 assembled instrument with the case, Nexus 7 tablet, and battery packs removed, and (d) fully assembled 451 4RP neutron spectrometer.

452 For the $\mathbf{4 R P}$ spectrometer, the goal was to utilize the same motherboard as the $\mathbf{6 C}$ but reduce the HDPE 453 mass (from that of the $\mathbf{6 C}$ ) by keeping only the portion separating the detector layers, resulting in a 454 rectangular prism geometry. In doing so, the total instrument weight dropped from $18.5 \mathrm{lbs}$., for the 6C, 455 to $13.8 \mathrm{lbs}$. for the $\mathbf{4 R P}$; the moderator portion of the $\mathbf{4 R P}$ weight was $3.4 \mathrm{lbs}$. Using the same 456 moderator radius/thickness optimization procedure, but with the definition given in section 2.3.1 457 (Figure 5 RIGHT) for the rectangular-prism radius, the optimal thickness of the HDPE for the 4RP 458 instrument was found to be approximately $2.75 \mathrm{~cm}$ to $3.0 \mathrm{~cm}$. Therefore, the same moderator thickness 459 as the $6 \mathrm{C}$ design, $2.29 \mathrm{~cm}$, could be used for the 4RP design without significant detriment to either 460 operational performance metric. Since reducing mass while retaining high intrinsic efficiency and 461 spectral sensitivity is critical to the commercial adoption of this type of instrument, it is necessary to 462 understand the tradeoffs between the $\mathbf{6 C}$ and $\mathbf{4 R P}$ moderator configurations. 
(a)
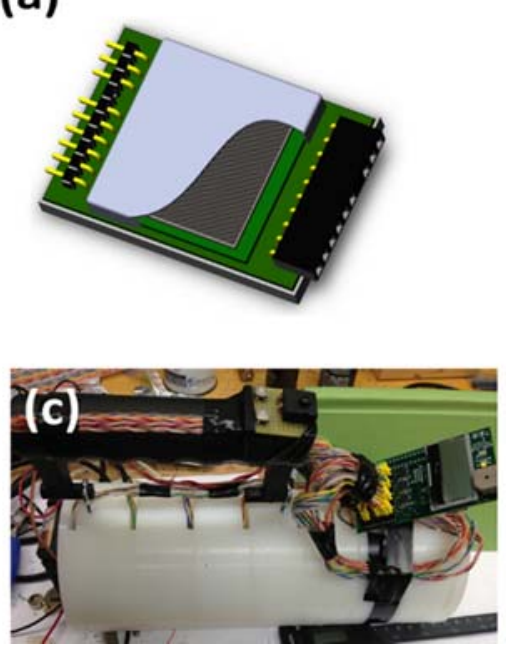

(b)
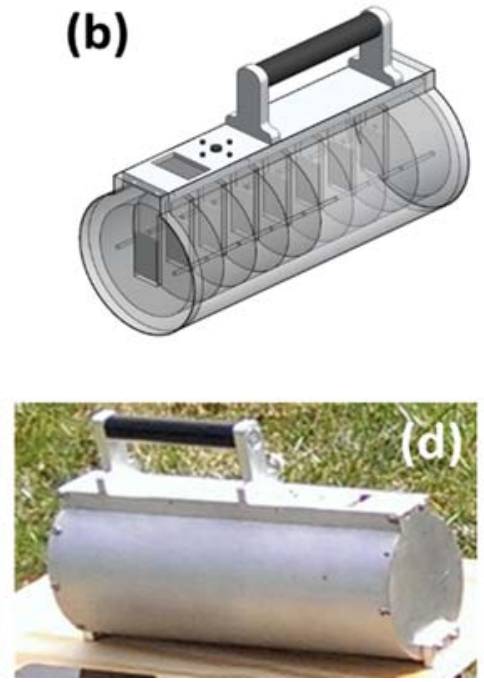

Figure 16. Photographs and renderings of the $4 \mathrm{C}$ system showing (a) an individual printed circuit board with single mounted microstructured neutron detector die, (b) assembly view showing the 8-MSNDs with recessed moderator slabs, motherboard, display, and case (transparent), (c) assembled instrument with the case, and battery packs removed, and (d) fully assembled $\mathbf{4 C}$ neutron spectrometer.

To consider even more dramatic tradeoffs in cost, mass, and complexity, the $\mathbf{4 C}$ moderating-type instrument was designed with a total weight restriction of $<9 \mathrm{lbs}$. and the use of only one 4-cm ${ }^{2}$ MSND (Figure 16a) at each of the eight depths into the moderator volume (Figure 16b). The same optimization process illustrated in Figure 14 was used to find the optimal radius and thickness combinations for the intrinsic efficiency and spectral sensitivity. The optimization process yielded a $5-\mathrm{cm}$ radius and $2.25-\mathrm{cm}$ thickness for optimal intrinsic detection efficiency, and a 5-cm radius and 3-cm thickness for optimal spectral sensitivity. A radius of $5.08 \mathrm{~cm}$ and thickness of $2.29 \mathrm{~cm}$ were used for the actual fabrication of the moderator slabs, based on practical considerations such as the availability of materials, and the total moderator weight was $5.0 \mathrm{lbs}$. Note that the optimal moderator dimensions for all design models were very similar, due to the predictable probabilistic nature of neutron energy loss and scattering angle via elastic scattering interactions in the common moderator material under consideration (HDPE). While moderator shape and detector board type played minor roles, the small differences in optimal configurations was largely due to the progressively restrictive weight constraints imposed from the $6 \mathrm{C}$ to the $\mathbf{4 R P}$ and $\mathbf{4 C}$ spectrometer designs.

\subsection{Intrinsic Efficiency ${ }^{1}$}

\footnotetext{
${ }^{1}$ All empirical intrinsic efficiency measurements reported here were collected with a bare ${ }^{252} \mathrm{Cf}$ source $100 \mathrm{~cm}$ from the instrument. Data was collected until the counting statistics for the overall instrument yielded less than $2 \%$ error. The instruments were positioned $120 \mathrm{~cm}$ above a concrete floor, $200 \mathrm{~cm}$ below a concrete ceiling, and $5 \mathrm{~m}$ from a single concrete wall facing the instrument to help minimize the asymmetry and overall detection of environmentally scattered neutrons. Finally, tests using a shadow shield validated that less than $5 \%$ of the detected neutrons were from environment scatter.
} 
The intrinsic neutron detection efficiency, for a particular energy spectrum, was defined in this work as the ratio of the number of neutrons detected to the number of source neutrons incident on the instrument moderator volume (Equation 1), represented as a percentage. This definition helps to normalize the results compared between instruments, as it partially accounts for the moderator dimensions. If the intrinsic neutron detection efficiency is reported for two or more faces of each instrument (e.g., front incidence and side incidence), a one-to-one comparison can be made between the detection systems. Further, one can use the intrinsic neutron detection efficiency to compute the absolute neutron detection efficiency for the source size and distance of interest to their application. This allows for direct comparisons between instruments with respect to the ability to passively detect neutron sources alone. To help put the values reported here in context, with an intrinsic efficiency to bare ${ }^{252} \mathrm{Cf}$ of $\sim 4.5 \%$ for neutrons incident on the front face of the $6 \mathrm{C}$ instrument (i.e., $182-\mathrm{cm}^{2}$ area), a $95 \%$ detection certainty of a 1E5-n/s source at 10-m distance could be made in 50 seconds.

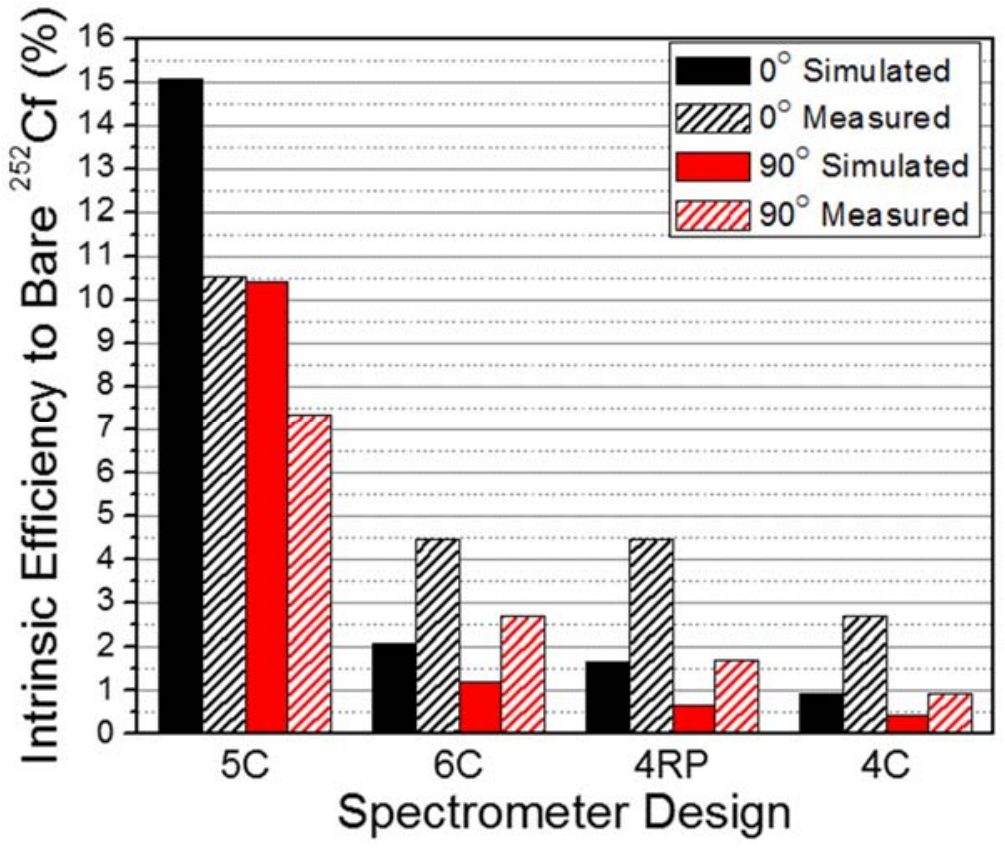

Figure 17. Simulated and measured intrinsic efficiency to bare ${ }^{252} \mathrm{Cf}$ (in \%) for each spectrometer model, from $0^{\circ}$ (front face) incidence and $90^{\circ}$ (side) incidence.

The simulated and measured intrinsic neutron detection efficiency values to bare ${ }^{252} \mathrm{Cf}$ for each instrument are compared in Figure 17 for front and side incidence $\left(0^{\circ}\right.$ and $90^{\circ}$ respectively). While the empirically measured intrinsic neutron detection efficiency values to bare ${ }^{252} \mathrm{Cf}$ for the $\mathbf{6 C}, \mathbf{4 R P}$, and $\mathbf{4 C}$ spectrometers are, on average, $1.7 \%$ greater ( $1.4 \%$ relative) than their simulated values from both the front and side, the measured value of $\mathbf{5 C}$ is $4.5 \%$ less ( $30.1 \%$ relative) than its simulated value from the front and $3.1 \%$ less (29.5\% relative) than its simulated value from the side. In the case of the $\mathbf{5 C}$ design, the MCNP simulations had to be significantly simplified due to the complexity of the detector electronics boards and did not account for the neutron capture properties of the FR-4 printed circuit board,

506 resulting in a greater efficiency in simulation than experiment. In the case of the $\mathbf{6 C}, \mathbf{4 R P}$, and $\mathbf{4 C}$, the 507 detector boards were simpler to model, allowing for the inclusion of the FR-4 PCBs and electronics in 
simulation, leading to the correct trends for these instruments, as shown in Figure 17. The relatively small differences in magnitude between the simulated and measured efficiencies in these cases are mostly due to environmental scattering effects which were not included in simulation. Environmental neutron scattering leads to an increase in the number of thermalized neutrons impinging on the spectrometer volume and, in turn, a greater intrinsic neutron detection efficiency. The $\mathbf{5 C}$ spectrometer achieves the highest overall efficiency values due to having a much larger total active detector area than the other instruments. For a more relative comparison, however, the instruments' intrinsic efficiency per $\mathrm{cm}^{2}$ of total detector area can instead be considered. Viewing the experimental results at $0^{\circ}$ (front face) incidence in this detector-area-normalized manner, the efficiency per unit detector area trends in the opposite direction; $0.00277 \%$ per $\mathrm{cm}^{2}$ for the $5 \mathrm{C}, 0.00876 \%$ per $\mathrm{cm}^{2}$ for the $6 \mathrm{C}, 0.00874 \%$ per $\mathrm{cm}^{2}$ for the $\mathbf{4 R P}$, and $0.0841 \%$ per $\mathrm{cm}^{2}$ for the $4 \mathrm{C}$. This demonstrates that although intrinsic detection efficiency was sacrificed to some degree with each design simplification (5C $\rightarrow \mathbf{6 C} \rightarrow \mathbf{4 R P} \rightarrow \mathbf{4 C}$, in order of decreasing complexity) due to decreased moderator volume and/or detector area, the design optimization processes (discussed in previous sections) for each instrument were successful in mitigating these sacrifices by maximizing efficiency under each increasingly restrictive set of design constraints.

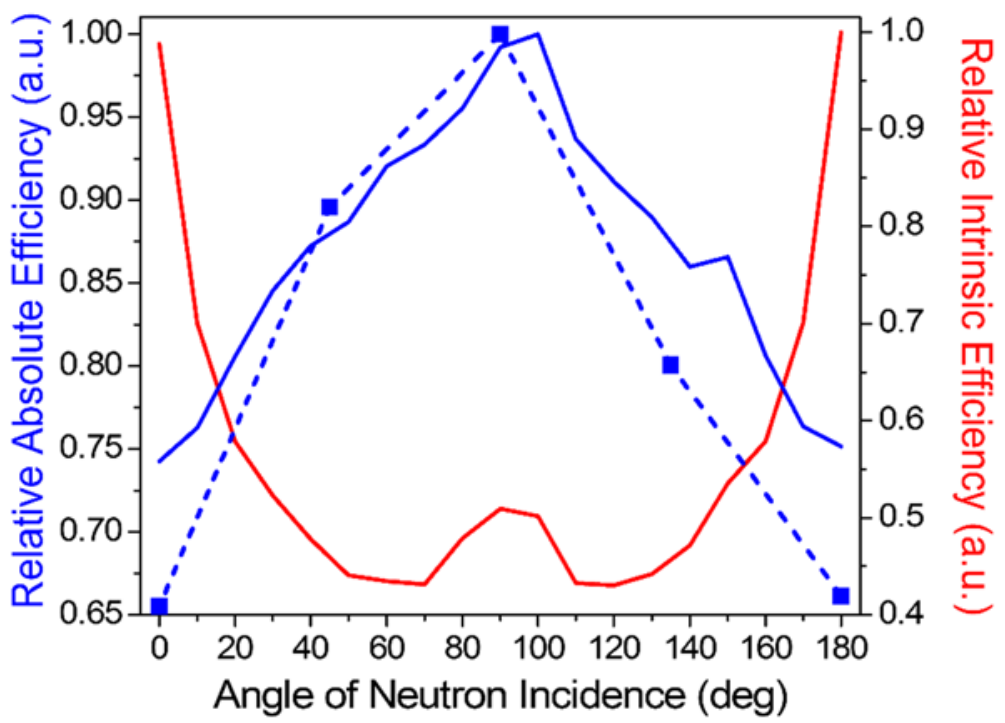

Figure 18. 5C spectrometer simulated relative intrinsic efficiency $(-)$, Simulated Relative Absolute Efficiency (-), and Experimental Relative Absolute Efficiency (- - ) to bare ${ }^{252} \mathrm{Cf}$, as a function of neutron incident angle (in degrees). All efficiencies are normalized to allow for direct comparison.

The simulated and measured angular dependence of the absolute neutron detection efficiency, and the simulated angular dependence of the intrinsic neutron detection efficiency, of the $\mathbf{5 C}$ design are shown in Figure 18, for bare a ${ }^{252} \mathrm{Cf}$ neutron source. In all cases, $0^{\circ}$ indicates normal incidence on the front face of the instrument. The reason the intrinsic neutron detection efficiency is highest at $0^{\circ}$ and $180^{\circ}$ is that, for each of the instruments, much of the latter half of the $30-\mathrm{cm}$ axial depth is used to thermalize incident neutrons from the ${ }^{252} \mathrm{Cf}$ source whose energy is in excess of $5 \mathrm{MeV}$. In the case of $\mathbf{5 C}$, the 12.7$\mathrm{cm}$ moderator diameter is not sufficient to thermalize the majority of side-incident neutrons $\left(90^{\circ}\right)$. 
535 Absolute detection efficiency is highest for side incidence as the total cross-sectional area is more than a 536 factor of 3 greater than the front face of the instrument. The low points in the intrinsic efficiency at $\sim 55^{\circ}$

537 and $\sim 125^{\circ}$ are due to the excess path length of FR4, and are only specific to the $\mathbf{5 C}$ design.

\section{$538 \quad 3.2 \quad$ Identification}

539 To identify sources of neutron radiation, the instrument software utilizes the previously discussed

540 Pearson product-moment cross-correlation coefficient (Equation 2). First, an extensive library of

541 spectrometer responses (summed detector board counts as a function of axial depth $N(z)$ ) must be

542 generated through either MCNP simulation or empirical measurement, and should include as many

543 neutron source types and moderator/absorber configurations as is necessary for a given application.

544 With this library of responses generated and uploaded to the spectrometer's software, live response

545 data collected from an unknown source is then compared to each library response using the Pearson

546 cross-correlation coefficient, $r$, and ranked to yield the closest matching source (i.e., the source type

547 with the most correlated library response is chosen as the most likely source identity). This process is

548 repeated and displayed to the user in one-second updates as the collected data accumulates.

549 To test the neutron source identification capabilities of the $\mathbf{6 C}$ and $\mathbf{4 R P}$ spectrometers under various

550 moderator/absorber configurations as a function of both time and distance from the source, a series of

551 experiments were planned and conducted in a large grass field (Figure 19, TOP) at the Naval Surface

552 Warfare Center Carderock Division in West Bethesda, Maryland. The neutron source used for the

553 experiments was a $4.432 \times 10^{5}-\mathrm{n} / \mathrm{s}^{252} \mathrm{Cf}$ source $(104.77 \mu \mathrm{Ci})$ inside a thin moderating NIST-RG-LL-09-

554 252-D sphere (Figure 19 A). 

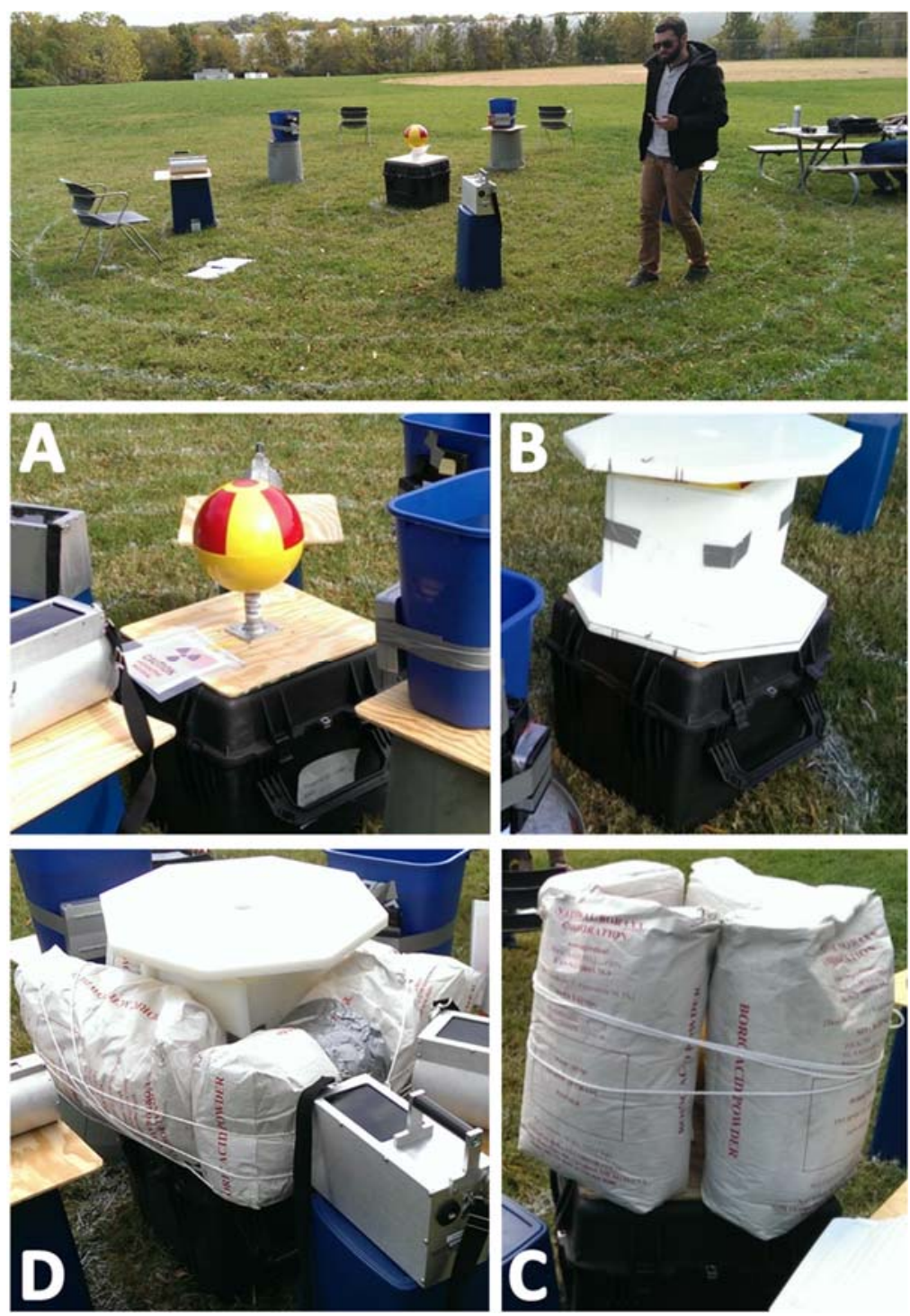

Figure 19. Neutron source identification experimental setup (TOP) with lines indicating radial distances

557 from the neutron source in 0.5-m increments. Neutron source + moderator/absorber configurations

558 tested: (A) $4.432 \times 10^{5} \mathrm{n} / \mathrm{s}^{252} \mathrm{Cf}$ source inside moderating NIST-RG-LL-09-252-D sphere, (B) 1" HDPE

559 surrounding (A), (C) Four 50 -lb sacks of $\mathrm{H}_{3} \mathrm{BO}_{3}$ tied around (A), and (D) Four 50-lb sacks of $\mathrm{H}_{3} \mathrm{BO}_{3}$ tied around (B).

Lines were marked in the grass to indicate radial distances from the neutron source in $0.5-\mathrm{m}$ increments,

563 from $0.5 \mathrm{~m}$ to $5.0 \mathrm{~m}$, as shown in Figure 19 (TOP). Measurements were then taken with both

564 instruments at each distance, with the data collection time adjusted to ensure less than $5 \%$ count error.

565 This set of experiments was then repeated 3 more times with additional moderator/absorber

566 configurations: 1" of high density polyethylene surrounding the NIST sphere (Figure 19, B), four 50-lb

567 sacks of $\mathrm{H}_{3} \mathrm{BO}_{3}$ (boric acid) tied around the NIST sphere (Figure 19, C), and 1" of HDPE plus four 50-lb

568 sacks of $\mathrm{H}_{3} \mathrm{BO}_{3}$ tied around the NIST sphere (Figure 19, D). A summary plot of the results of these 
experiments is shown in Figure 20 below. Neutron source libraries included simulated approximations of configurations A-D as well as bare and moderated variants of americium-beryllium (AmBe), plutoniumberyllium (PuBe), and americium-lithium ( $\mathrm{AmLi}$ ).

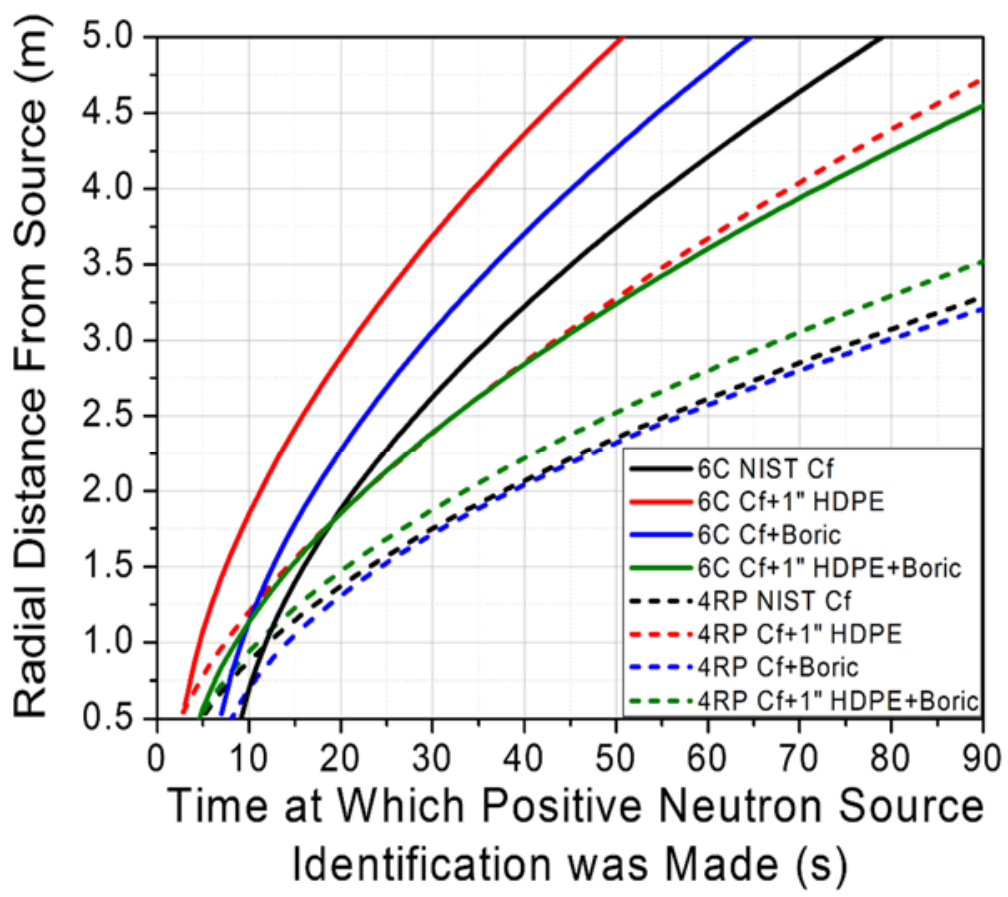

Figure 20. Identification experiment summary plot. The time at which a positive neutron source + moderator/absorber identification was made (in seconds) is plotted as a function of radial distance from the neutron source (in meters).

As shown in the summarized empirical results of Figure 20 , the $\mathbf{6 C}$ spectrometer consistently outperforms the 4RP spectrometer in positively identifying neutron sources in less time. In the case of the $\mathbf{6 C}$ spectrometer (solid lines in Figure 20 ), the experimental results may seem surprising upon initial inspection, as two of the source configurations with additional moderator/absorber (configurations B and $\mathbf{C}$ in Figure 19, solid red and blue lines in Figure 20 respectively) were positively identified in less time than the lightly moderated configuration with the NIST sphere alone (configuration A in Figure 19, solid black line in Figure 20). This result is informative in that it highlights the pragmatically significant relationship between the instrument's intrinsic efficiency and the correlation coefficient method of neutron source identification. In order to achieve a positive neutron source ID in the shortest amount of time with the correlation coefficient method, a high count rate is needed to provide sufficient counting statistical accuracy in the shortest amount of time. Since the $\mathbf{6 C}$ spectrometer is most efficient to neutrons at the bottom end of the fast neutron energy range, the instrument achieved the highest count rate from the source configuration with an additional 1" of HDPE (higher amount of neutron moderation, configuration B), and identified this configuration in the shortest amount of time for all distances measured. The $6 \mathrm{C}$ achieved the next highest count rate from the configuration with $\mathrm{H}_{3} \mathrm{BO}_{3}$ (boric acid) surrounding the NIST sphere (configuration $\mathbf{C}$ ) and yielded the next best identification results. This relatively high count rate is due to the additional moderation via elastic scattering with 
hydrogen atoms in the boric acid. However, this effect is partially counteracted by moderately high energy neutron absorptions by ${ }^{10} \mathrm{~B}$ atoms (also present in boric acid) at these energies, despite the reduced cross-section. Configuration D, with both 1" of HDPE and boric acid surrounding the NIST sphere, yielded the lowest count rate in the $\mathbf{6 C}$ spectrometer. This relatively low count rate is due to the fact that many of the high energy neutrons exiting the NIST sphere are sufficiently moderated by the 1" of HDPE surrounding the sphere to have a high probability of subsequent absorption in the boric acid surrounding the HDPE. However, despite this heavy moderation and absorption, the $6 \mathrm{C}$ instrument was still able to positively identify the neutron source in less than 90 seconds at a distance of $4.5 \mathrm{~m}$. Although similar trends can be seen in the 4RP spectrometer results, the aforementioned effects are skewed due to this spectrometer's greater sensitivity to environmental scattering effects. In other words, neutrons scattered off the ground are detected after entering the instrument from the bottom, decreasing the statistical predictability of the axial count distribution $\left(N_{A}(z)\right.$ in Equation 2$)$ on which the correlation coefficient method of neutron source identification is based.

Although the identification studies were primarily designed to test the correlation coefficient methodfor the $\mathbf{6 C}$ and $\mathbf{4 R P}$ spectrometers - against challenges inherent to non-proliferation (e.g., scattering from operational environments), high certainty identification of neutron sources in real time (as shown in Figure 20) also has impact in more accurately measuring neutron dose. Specifically, determining the identity of a neutron source and its flux allows for convolution of the exact energy spectrum and fluence with the ICRP 74 standard to yield an accurate measure of the ambient neutron dose equivalent [23]. This method overcomes the deficiencies of the 'linear combination of responses' method presented by Oakes et al. [1]. Alternatively, given the unique and numerous response functions provided by these moderating type neutron spectrometers, the absolute neutron energy and its fluence can also be determined

616 indirectly using unfolding techniques. Reports describing further details of the neutron dose equivalent and application of unfolding methods to the instruments described herein will follow this work.

\subsection{Conclusion}

Four solid-state, moderating-type neutron spectrometers were designed, fabricated, and tested in an effort to improve on the neutron source detection and identification capabilities of long counters and Bonner-sphere-based instruments while making them portable. Through moderator geometry optimization, it was found that an instrument with a cylindrical moderator geometry can achieve a greater intrinsic neutron detection efficiency and greater energy sensitivity than an instrument with a rectangular prism geometry of the same weight. In studying the effects of individual MSND thermal neutron detection efficiency on overall instrument performance, it was shown that increasing the MSND thermal neutron detection efficiency above $22 \%$ provides diminishing returns to an instrument's intrinsic efficiency to bare ${ }^{252} \mathrm{Cf}$. Through theoretical examination of the ratio between an instrument's outer moderator radius and outer detector radius, an outer detector radius of $3.8 \mathrm{~cm}$ and outer moderator radius of $7.8 \mathrm{~cm}$ (to reflect outbound neutrons) was found to be optimal for a cylindrical instrument of approximately $15 \mathrm{lbs}$. After investigation of various moderator materials' effects on operational performance metrics, it was shown that increasing the hydrogen concentration of the moderator material drastically increases an instrument's intrinsic neutron detection efficiency and energy sensitivity. In this materials study, high density polyethylene was found to be more optimal for a 
wider range of applications than the other materials tested. Of the four fabricated spectrometers, the $\mathbf{5 C}$ design exceeds the others in every category of operation, but is the most complex, the most costly to build, and the most physically cumbersome to operate. The $\mathbf{6 C}, \mathbf{4 R P}$, and $\mathbf{4 C}$ designs were optimized and built to address the deficiencies of the $\mathbf{5 C}$ design while simultaneously maximizing their individual operational performance, under increasingly restrictive cost and weight constraints, in order to mitigate the unavoidable tradeoffs associated with decreases in moderator volume and/or active detector area. These four instruments are the first representatives of a new class of hand-held, semiconductor-based, moderating-type neutron spectrometers of varying cost, weight, and design complexity, that provide a means to high intrinsic detection efficiency and neutron source identification through real-time energysensitive measurements of free neutron fields, ranging from thermal energies to the high end of the evaporation spectrum.

\section{Acknowledgements}

This work was funded by the Office of Naval Research, award nos. N00014-11-1-0157 and N00014-13-10402, and by the Defense Threat Reduction Agency, award no. HDTRA1-14-P-005. ANC wishes to thank Mark Strickman in performing the SWORD simulations.

\section{References}

[1] Thomas M. Oakes, S.L. Bellinger, W.H. Miller, E.R. Myers, R.G. Fronk, B.W. Cooper, T.J. Sobering, P.R. Scott, P. Ugorowski, D.S. McGregor, J.K. Shultis, A.N. Caruso, "An Accurate and Portable Solid State Neutron Rem Meter", Nuclear Instruments and Methods in Physics Research Section A: Accelerators, Spectrometers, Detectors and Associated Equipment 719 (2013) 6-12.

[2] Richard L. Bramblett, R.I. Ewing, and T.W. Bonner, "A New Type of Neutron Spectrometer", Nuclear Instruments and Methods 9.1 (1960) 1-12.

[3] Walter J. McNeil, S.L. Bellinger, T.C. Unruh, C.M. Henderson, P. Ugorowski, B. Morris-Lee, R.D. Taylor, D.S. McGregor. "1-D array of perforated diode neutron detectors", Nuclear Instruments and Methods in Physics Research Section A: Accelerators, Spectrometers, Detectors and Associated Equipment 604.1 (2009) 127-129.

[4] A.O. Hanson, J. L. McKibben, "A Neutron Detector Having Uniform Sensitivity from $10 \mathrm{keV}$ to 3 MeV", Physical Review 72.8 (1947) 673.

[5] Y. Tanimura, J. Saegusa, M. Yoshida, "Design of a single moderator-type neutron spectrometer with enhanced energy resolution in the energy range from a few to $100 \mathrm{keV}$ ", Nuclear Instruments and Methods in Physics Research Section A: Accelerators, Spectrometers, Detectors and Associated Equipment 547.2 (2005) 592-600.

[6] Hiroki Tsuji, S. Maeda, H. Tomita, J. Kawarabayashi, T. Iguchi, T. Matsumoto, J.I. Hori, "Epithermal Neutron Response on Moderated Neutron Spectrometer with Multi-Resonance Filters", Prog. Nucl. Sci. Technol. 1 (2011) 316-319. 
669 [7] Andrew C. Stephan, V. D. Jardret, Neutron Detector, United States Patent no. 7514,694 (2009).

670 [8] Anthony N. Caruso, "The Physics of Solid-State Neutron Detector Materials and Geometries", $671 \quad$ Journal of Physics: Condensed Matter 22 (2010) 443201.

672 [9] IAEA, "Compendium of Neutron Spectra and Detector Responses for Radiation Protection

673

674

675

676

677

678

679

680

681

682

683

684

685

686

687

688

689

690

691

692

693

694

695

696

697

698

699

700

701 Purposes", Technical Reports Series no. 403, Supplement to Technical Reports Series No. 318. Vienna (2001).

[10] Marcel Reginatto, P. Goldhagen, S. Neumann, "Spectrum Unfolding, Sensitivity Analysis and Propagation of Uncertainties with the Maximum Entropy Deconvolution Code MAXED", Nuclear Instruments and Methods in Physics Research Section A: Accelerators, Spectrometers, Detectors and Associated Equipment 476.1 (2002) 242-246.

[11] Marcel Reginatto, "What can we learn about the spectrum of high-energy stray neutron fields from Bonner sphere measurements?", Radiation Measurements 44.7 (2009) 692-699.

[12] Karl Pearson, "Note on Regression and Inheritance in the Case of Two Parents", Proceedings of the Royal Society of London 58 (1895) 240-242.

[13] J.K. Shultis, D.S. McGregor, "Design and Performance Considerations for Perforated Semiconductor Thermal-Neutron Detectors," Nuclear Instruments and Methods in Physics Research A: Accelerators, Spectrometers, Detectors and Associated Equipment 606 (2009) 608636.

[14] Steven L. Bellinger, R.G. Fronk, T.J. Sobering, D.S. McGregor, "High-Efficiency Microstructured Semiconductor Neutron Detectors that are Arrayed, Dual-Integrated, and Stacked", Applied Radiation and Isotopes 70.7 (2012) 1121-1124.

[15] Marcel Reginatto, "Resolving Power of a Multisphere Neutron Spectrometer", Nuclear Instruments and Methods in Physics Research Section A: Accelerators, Spectrometers, Detectors and Associated Equipment 480.2 (2002) 690-695.

[16] Brian W. Cooper, S.L. Bellinger, A.N. Caruso, R.G. Fronk, W.H. Miller, T.M. Oakes, J.K. Shultis, T.J. Sobering, D.S. McGregor, "Neutron Energy Spectrum with Microstructured Semiconductor Neutron Detectors", Nuclear Science Symposium and Medical Imaging Conference (NSS/MIC), IEEE (2011) 4783-4786.

[17] Mark J. Harrison, Q. Cherel, M. Monterial, "Design of a Moderated Multidetector Neutron Spectrometer for Optimal Specificity", Nuclear Science Symposium and Medical Imaging Conference (NSS/MIC), IEEE (2011) 4787-4794.

[18] Steven L. Bellinger, A.N. Caruso, B.W. Cooper, R.G. Fronk, C.B. Hoshor, D.S. McGregor, W.H. Miller, E.R. Myers, T.M. Oakes, B.J. Rogers, P.B. Ugorowski, J.K. Shultis, S.M. Young, "Apparatuses and 
Methods for the Localization and Identification of Neutron Sources and/or Neutron Shielding Materials and Neutron Dose Equivalent", USPTO No. 9,081,100 issued 14 July 2015.

[19] Paul Goldhagen, "An Extended-Range Multisphere Neutron Spectrometer with High Sensitivity and Improved Resolution", Nuclear Technology: Radiation Measurements and General Instrumentation 175.1 (2011) 81-88.

[20] Steven L. Bellinger, R. G. Fronk, W. J. McNeil, T. J. Sobering, D. S. McGregor, "Improved HighEfficiency Stacked Microstructured Neutron Detectors Backfilled with Nanoparticle LiF," IEEE Transactions on Nuclear Science 59.1 (2012) 167-173.

[21] Simon R. Bolding, "Design of a Neutron Spectrometer and Simulations of Neutron Multiplicity Experiments with Nuclear Data Perturbations", Master of Science Thesis, Kansas State University, Manhattan, Kansas (2013).

[22] Thomas M. Oakes, "Modeling and Analysis of a Portable, Solid-State Neutron Detection System for Spectroscopic Applications", Doctor of Philosophy Dissertation, University of Missouri, Columbia, Missouri (2012).

[23] Andrea Saltos, "Cross Correlation Calculations and Neutron Scattering Analysis for a Portable Solid State Neutron Detection System", Master of Science Thesis, University of Missouri, Columbia, Missouri (2015). 